\title{
Esprits et possession
}

Si les šêdîm n'apparaissent pas souvent dans la Bible hébraïque, il y a néanmoins une autre catégorie d'agents que l'on rencontre plus fréquemment et qui pourrait être rapprochée assez étroitement des démons, à savoir celle de rûhōt, $\pi v \varepsilon \hat{\mu} \mu \alpha \tau \alpha$ en grec. Comme déjà observé par Daniel Barbu, rûah représente à bien des égards la notion indigène qui se rapproche le plus de la catégorie moderne de « démon », notamment car la modalité d'action des esprits est davantage la possession ${ }^{1}$. La manière dont les esprits s'emparent des humains est particulièrement évidente dans les livres historiques (notamment Juges, Samuel et Rois) ainsi que dans le livre d'Ézéchiel. À cet égard, nous retiendrons le texte décrivant la scène de conseil divin dans le premier livre des Rois, au chapitre 22, lorsque le prophète Michée a une vision de l'assemblée qui se tient devant Yhwh : pendant l'assemblée, un des esprits de la cour céleste prend la parole et s'offre comme volontaire pour devenir un esprit de mensonge dans la bouche des prophètes qui entourent la cour du roi Achab à Samarie. Ce texte est d'autant plus intéressant pour mon propos qu'une des versions de la LXX, dite «lucianique», contient des informations qui ne sont pas présentes dans le texte massorétique. Mais l'exemple le plus fameux de possession par les esprits se trouve probablement dans le récit de l'arrivée du jeune David à la cour du roi Saül dans le premier livre de Samuel (chapitres 16-19) où David, en vertu de ses capacités de musicien, soulage à plusieurs reprises le roi qui était tourmenté par un mauvais esprit. En outre, les esprits, notamment ceux impurs, sont souvent associés aux démons dans plusieurs traditions du Second Temple en grec et en hébreu, à la fois dans les traditions hénochiques et dans les écrits de Qumrân ${ }^{2}$. Enfin, esprits impurs et démons sont également identifiés dans le Nouveau Testament ${ }^{3}$. C'est donc à la notion d'esprits et à leurs rôles dans la LXX que ce chapitre sera dédié. Pour comprendre le fonctionnement des esprits dans la LXX il faut d'abord revenir sur deux points préliminaires et plus généraux. Le premier concerne la sémantique des mots rûah et $\pi v \varepsilon \hat{\mu} \mu \alpha$ en hébreu et en grec qui a fait l'objet d'un grand nombre d'études dans le cadre

1 Barbu et Rendu-Loisel 2009, p. 307.

2 La bibliographie à ce sujet est vaste. Pour un bilan récent avec bibliographie à jour, on peut voir Stuckenbruck 2014 b et Tigchelaar 2018.

3 Voir, par exemple, Lc 4,33; 9,42; Ap 16,13-14. Sur l'importance des esprits impurs dans le Nouveau Testament on peut voir, entre autres, Wahlen 2004. 
de l'Ancien et du Nouveau Testament; le deuxième, qui a reçu une attention moindre, aborde la relation entre l'esprit de Yhwh et les autres esprits, dans la Bible hébraïque et dans la Lxx.

\section{Les esprits entre nature et psychologie}

Les nombreuses études sur rûah ainsi que les principaux dictionnaires assignent une signification primaire d' ' air en mouvement», et donc de « vent», conformément à sa dérivation de la racine $r w h$, «souffler» ${ }^{4}$. De cette signification dérive une deuxième valeur de «souffle», «haleine» et, par métonymie, de «vie», «principe vital $»^{5}$. La force vitale représentée par rûaḥ est propre autant aux hommes qu'aux animaux ${ }^{6}$ : en ce sens rûah est en relation avec un autre terme qui indique en hébreu la vie et l'âme, nepeš, et se définit en partie par rapport à celui-ci. D'après Hans Wolff, les deux termes peuvent être parfois utilisés comme synonymes, comme par exemple en Is 26,9, Job 12,10 (esprit et âme humaines) et Is 42,1 (esprit et âme divins). D'autres exemples suggèrent que lorsque nepeš met l'accent sur l'organe de la respiration (avec parfois la signification de «gorge»), l'accent de rûah est plutôt mis sur le mouvement d'air qui se produit pendant le processus : un bon exemple en ce sens se trouve en Jer 2,24, qui décrit le hennissement d'une ânesse sauvage en combinant les deux termes ${ }^{7}$. Mais la différence fondamentale concernant l'extension sémantique des deux termes est probablement à reconnaître dans le fait que la nepeš appartient exclusivement aux hommes et aux animaux tandis que la rûah ${ }^{8}$ est un attribut lié davantage à la divinité et qui peut être transmis du dieu à l'homme, soit sous forme de principe vital, soit sous celle d'une inspiration particulière et temporaire ${ }^{9}$. Rûah joue un rôle important dans le contexte de

4 Une exception en ce sens est représentée par Henri Cazelles (1982), qui considère la racine $r w h$ comme ayant à l'origine un sens spatial («être ample», «être large») et voit donc en rûaḥ le sens primaire d' « espace », « largeur ». Bien que l'air en mouvement inclue également une dimension spatiale, une lecture de toutes les occurrences selon cette signification me parait forcée. Voir également les remarques de Albertz et Westermann 1997, p. 1498.

5 Voir, par exemple, Schoemaker 1904; Lys 1962; Albertz et Westermann 1997; Tengström 2004; Wolff 2010, p. 64-74; Blischke 2019, p. 1-5.

6 Voir déjà Gen 7,15 et 22.

7 Une interprétation similaire est possible pour Job 7,11.

8 En hébreu biblique, nepeš est toujours féminin ; rûaḥ est au féminin dans la grande majorité des cas (tout comme les noms des vents), même si dans un groupe restreint d'attestations le substantif se trouve au masculin : voir Joüon $\S 134, h$ et $k$; нALOT, s.v. rûah.

9 Voir, à ce sujet, récemment Feldmeier et Spieckermann 2011, p. 206-221, Blischke 2019, p. $289-298$. 
l'inspiration prophétique, mais elle a également d'autres domaines d'action: l'habilité technique extraordinaire des artisans de la tente de la rencontre en Exode est dirigée par un esprit de sagesse et d'intelligence pratique ${ }^{10}$; la puissance guerrière dans les livres de Josué et des Juges est également un produit de l'esprit ${ }^{11}$; enfin, comme nous le verrons plus en détail par la suite, la présence ou l'absence de l'esprit divin joue un rôle significatif dans l'élection royale. Il est, en revanche, moins évident de savoir si rûah est toujours à comprendre comme une puissance distincte de la divinité ou s'il est parfois conçu comme un attribut divin, en quelque sorte inséparable de Yhwh lui-même et lié en dernière instance à son souffle, comme cela semble être suggéré par quelques psaumes où l'esprit est mis en parallèle avec la «face» de la divinité : «Où j'irai, loin de ton esprit? où je m'enfuirai, loin de ta face? $»^{12}$. La question se complexifie encore par le fait que, dans la Bible hébraïque, d'autres esprits sont mentionnés à côté de rûaḥ yhwh et de rûaḥ ělōhîm. Par conséquent, même en acceptant que les esprits soient toujours conçus comme des puissances divines, il faut encore se demander s'il s'agit d'agents individualisés au service du dieu ou, plutôt, d'une fonction, au sein de la cour céleste, qui peut être occupée par différents personnages.

Des concepts comparables à la rûah biblique existent ailleurs au ProcheOrient ancien. En accadien, le vocabulaire lié aux vents et aux esprits a parfois été rapproché de rûaḥ. Les termes les plus pertinents en ce sens sont šaru, «vent», «air», «souffle», et šehu/šehanu, «vent», «souffle», qui indique également une émanation spécifiquement liée à la possession et à la transe prophétique $^{13}$. En outre, dans la mythologie ouest-sémitique les vents, désignés par le terme $r w h$, font également partie de l'entourage du dieu Baal en tant que dieu de l'orage ${ }^{14}$. La signification de rûah comme « souffle de vie » est également attestée dans l'épigraphie araméenne et punique ${ }^{15}$. En revanche, la

\footnotetext{
$10 \quad$ Ex 28,$3 ; 31,3$ et 5 .

11 Voir déjà Deut 34,9.

12 Ps 139,7. Voir également Ps 51,13; 104,29-30, et, à ce sujet, MacDonald 2013 qui voit dans l'esprit de Yhwh une forme de la présence divine particulièrement développée à l'époque postexilique. Il me semble que le rapprochement entre esprit et face de Yhwh est déjà suggéré en Gen 3,8.

13 CAD 17/2, s.v. ; Nissinen 2017, p. 175 qui cite une équivalence entre mahh $\hat{u}$, «prophète », et šēhānnu, «possédé » dans un commentaire néo-assyrien sur une série de présages Šumma $i z b u$. Pour une comparaison entre les «vents»/« esprits» mésopotamiens et bibliques, voir Tengström 2004, p. 370-371; récemment Lilly 2016 a et 2016 b. Les travaux de Nissinen (entre autres 2010 et 2017) sont la meilleure introduction au traitement comparatiste de la possession prophétique au Proche-Orient ancien, en Israël et en Grèce.

$14 \quad$ KTU 1.5.V.7.

15 Sfire III (KAI 224, l. 2); Carthage (KAI 79, l. 10-11).
} 
seule attestation connue de l'invocation d'un esprit «magique » qui soit indépendante des traditions bibliques est une incantation nabatéenne datant de 100 av. n. è.: le sorcier y invoque l'esprit en tant que puissance capable d'intervenir contre un maléfice ${ }^{16}$. Par ailleurs, les fréquentes mentions des esprits sur les bols d'incantations araméennes dépendent largement des traditions bibliques ${ }^{17}$. Quant aux documents puniques qui attesteraient de la présence des esprits, ils sont tous de lecture incertaine, voire très douteuse ${ }^{18}$. La Bible hébraïque apparait, donc, comme le contexte principal dans lequel l'action des esprits est décrite avec un certain détail.

Dans la Bible hébraïque la relation étroite du rûaḥ avec la vie de l'individu se développe ultérieurement dans une direction psychique qui ne trouve pas de véritable équivalent au Proche Orient antique : rûạ̣ désigne ainsi l'esprit comme siège des sentiments et des émotions, mais également des facultés intellectuelles. Il indique alors une disposition de lâme, une inclination émotionnelle, une attitude ou une manifestation de la volonté : des exemples de cet usage sont réunis dans le tableau 6 (p. 234).

Nous pourrions, de manière simplifiée, décomposer la sémantique de rûạ en trois volets interdépendants : un naturaliste, un divin et un psychologique. Une approche anthropologique permet d'affiner l'analyse linguistique concernant la relation entre ces trois aspects. En effet, la recherche anthropologique récente a démontré que le recours aux esprits pour décrire des émotions n'est pas surprenant dans un contexte antique. Pour Charles Taylor, la relation de l'individu avec l'environnement est caractérisée, à l'époque prémoderne, par un soi poreux ( "porous self $»^{19}$ ) en contraste avec le soi enveloppé («buffered») ou isolé («bounded») de l'époque moderne. Dans cette perspective, les émotions intenses ainsi que les conditions qui produisent un changement d'état ou d'esprit dans l'individu ne sont pas considérées comme un produit de son intériorité, mais comme ayant leur origine hors de la personne et pouvant la saisir de l'extérieur. Par conséquent, les limites de la physicalité de l'individu sont perçues comme perméables non seulement aux esprits et aux démons, mais aussi, de manière plus générale, aux forces cosmiques et aux agents naturels. Toutefois, cette différence de perception de la corporéité individuelle se retrouve au sein même de l'époque moderne. En effet, en comparant la

\footnotetext{
16 Le texte a été publié par Naveh 1979 : l'éditeur souligne effectivement le manque de parallèles proche-orientaux par rapport aux formules incantatoires inscrites sur ce caillou.

17 Pour un premier répertoire, voir Naveh et Shaked 1998.

18 Voir DNWSI, s.v. $r h_{l}$. La possible lecture de $r h t$ comme «esprits» en $C I S$ I, 6o68, l. 4 est refusée par Clermont Ganneau (RES 18, p. 19 et 22); Donner et Röllig (KAI 89); Amadasi 2003, p. 27-28; Schmitz 2012, p. 71.

Taylor 2007, 29, 33-41.
} 
construction du corps en Occident avec celle des sociétés dites traditionnelles, on observe une différence fondamentale dans la manière de concevoir les relations entre l'individu et le monde extérieur ainsi que dans la manière de percevoir le rapport entre esprit et corps. À cet égard il est particulièrement significatif que l'anthropologie adopte souvent les phénomènes de possession et de maladie mentale comme point d'observation privilégié pour la mise en perspective de telles différences dans la conception plus générale des rapports entre l'individu, la représentation du soi et le monde externe ${ }^{20}$.

Un excellent exemple du lien étroit entre représentation de la nature et physiologie humaine est offert par la médecine mésopotamienne, dans laquelle sont considérés comme agents responsables de maladies les démons, mais également les vents nocifs qui peuvent pénétrer dans le corps ${ }^{21}$. Dans le domaine des études bibliques, plusieurs recherches ont, désormais, souligné l'anachronisme et la non-pertinence de toute perspective qui sépare strictement les aspects cosmologiques, ou naturalistes, des aspects psychologiques dans le traitement des esprits. Comme le montre Carol Newsom, le phénomène d'après lequel le développement de l'identité personnelle est verbalisé et, donc, représenté en termes d'agentivité externe est particulièrement évident dans les textes de Qumrân, qui laissent une large place aux esprits ${ }^{22}$. Dans des textes tels que les Hodayoth ou celui que l'on appelle couramment le «Traité des deux esprits », les esprits sont un instrument souvent utilisé pour exprimer un conflit interne à l'individu. Dans la mesure où le dualisme cosmologique joue un rôle assez important dans l'anthropologie qumrânienne, ce phénomène n'est pas surprenant. Mais le dualisme n'est pas le seul cadre épistémologique dans lequel peuvent s'inscrire ce type de représentations. En suivant la piste de Newsom, Reed Carlson a soutenu encore récemment que le

20 Voir, par exemple, Stanley Tambiah (1990, p. 133-135) qui compare le traitement ayurvédique de la maladie mentale avec les pratiques de la médecine moderne occidentale, ainsi que les essais sur la possession en Afrique, Mélanésie et Papouasie-Nouvelle-Guinée réunis en Lambek 1998. Reconnaître que le dualisme entre corps et esprit est un produit de la philosophie et de la science européenne de l'époque moderne, et donc qu'il est historiquement et culturellement déterminé, ne signifie pas pour autant nier l'universalité de la perception du soi ni la conscience d'une séparation entre un soi «interne » ou immatériel et un soi «physique », comme le rappelle justement Philippe Descola (2005, p. 210-226). D'après Descola il s'agit plutôt d'une « variante locale d'un système plus général de contrastes élémentaires » que chaque société élabore à sa manière (ibid., p. 219).

21 Voir, par exemple, Scurlock 2006, no. 91, 1. 13; 115, l. 33 ; 186, 1. 2-3; Geller 2007 a, no. 12, 1. 13-32 ; Pour la Bible hébraïque, on peut voir les études de Lilly 2016 a, 2016 b.

Newsom 2017. 
langage des esprits est une composante essentielle à la construction de l'identité personnelle déjà dans la Bible hébraïque ${ }^{23}$.

En partant de ces présupposés, plusieurs questions se posent quant à la manière de concevoir les esprits divins. Il faudrait, d'abord, se demander si le recours aux esprits comme vecteurs de forces psychiques est à mettre en relation avec leur représentation comme entités autonomes, ou du moins comme des puissances occupant une fonction bien définie au sein de la cour céleste, telle qu'elle est décrite dans certains passages bibliques, ou s'il s'agit de deux usages complètement distincts. D'autres questions centrales concernent l'autonomie des esprits par rapport à l'autorité de Yhwh ainsi que la possibilité d'une différenciation entre l'esprit de Yhwh et les autres esprits. En outre, étant donné que dans l'Antiquité l'externalisation du soi est un phénomène documenté pour les hommes, mais aussi pour les dieux, il faudrait, afin de comprendre la place des esprits dans l'«anthropologie des émotions », clarifier les relations existantes entre l'expression des sentiments et les facultés cognitives chez les hommes ainsi que chez les dieux. Il faudrait, ensuite, évaluer la traçabilité des développements de la conceptualisation des esprits à l'intérieur même de la Bible hébraïque ainsi que dans la Lxx par rapport à la Bible hébraïque. L'ampleur de l'enquête sort alors largement des limites de la recherche sur le démoniaque dans la LXX : certaines questions sont, par conséquent, destinées à demeurer ouvertes ici. Dans les pages qui suivent, j'essaierai, néanmoins, de jalonner quelques pistes de recherche.

\subsection{L'esprit et les esprits}

Un premier problème, concernant à la fois la Bible hébraïque et la LXX, est la délimitation du corpus: autrement dit, comment définir les critères permettant d'identifier les passages où les esprits sont décrits comme des puissances agissantes? Certes, la majorité des chercheurs s'accorde pour voir des puissances en action en $1 \mathrm{R} 22$ et $1 \mathrm{~S} 16-19$ bien que, respectivement à chaque livre, les catégories insatisfaisantes de «personnification» de la notion de mensonge et «d'objectivation» de la maladie de Saül soient parfois encore employées pour décrire les esprits ${ }^{24}$; toutefois se pose la question de savoir si ces épisodes sont à interpréter comme des cas exceptionnels, et donc isolés, ou s'ils sont paradigmatiques d'une manière plus générale de comprendre la

23 Carlson 2019.

24 Voir respectivement H.-P. Müller 1999, p. 325 ; McCarter 1999, p. 319-320, justement critiqués par Hamori 2010, p. 15-16. Ernst Axel Knauf (2019, p. 505) donne une interprétation encore différente et théologisant de l'esprit d'1 R 22, car il le considère comme une « forme d'émanation physique de Yhwh» qui aurait ici évolué vers une «personne autonome agissante », dans une sorte d'anticipation trinitaire. 
nature et le fonctionnement des esprits, notamment grâce aux descriptions plus exhaustives de ces récits.

Trois études récentes se sont penchées sur le sujet. Dans un article de 2010, Esther Hamori adopte des critères thématiques et narratologiques pour séparer les occurrences où rûah indique seulement une inclination, « un état d'esprit » pour ainsi dire, de celles qui font plus précisément référence à une puissance céleste. Elle sélectionne plusieurs contextes dans lesquels Yhwh envoie un esprit dont l'action a pour but de transmettre aux humains un faux message ou, plus généralement, de les tromper: le résultat final est la mort de la personne affectée par l'esprit ${ }^{25}$. Les passages principaux analysés par Hamori sont le conseil divin en $1 \mathrm{R} 22$, les références à l'esprit mauvais dans l'histoire de Saül et David dans le premier livre de Samuel (chapitres 16-19), l'esprit mauvais qui déclenche un conflit entre Abimelech et les habitants de Sichem en Jug 9,2324 et la mauvaise inspiration suscitée par Yhwh au roi d'Assyrie qui retourne alors dans son pays, où il sera tué $(2 \mathrm{R} 19,7)^{26}$. D'après Hamori, il existerait une tradition ancienne concernant un esprit de fausseté, différent de l'esprit de Yhwh, qui serait préposé à des buts trompeurs: Yhwh se servirait de cet esprit au lieu du sien à chaque fois qu'il doit envoyer un message pernicieux. Même si l'idée que la fonction principale des esprits serait la transmission de faux messages n'émerge pas avec la même clarté dans tous les textes pris en compte ${ }^{27}$, le mérite d'Hamori est d'avoir montré que $1 \mathrm{R} 22$ n'est pas à considérer comme un cas isolé ni comme une exception, mais qu'il s'inscrit dans une tradition plus large d'esprits divins subordonnés à la divinité qui interviennent auprès des hommes.

Dans son travail sur le conseil divin, Ellen White refuse l'idée que tous ces textes fassent référence au même esprit. Elle ne comprend d'ailleurs pas l'esprit comme une entité individualisée, mais comme une position au sein de la cour céleste : le caractère fonctionnel serait alors signalé par la présence de l'article (härûah) qui n'apparaît que dans la scène de conseil céleste d'1 R 22

25 Hamori 2010.

26 Elle envisage, en outre, une série de passages secondaires qui font allusion au même motif: l'esprit de torpeur versé par Yhwh sur les prophètes en Is 29,9-10 ; la vision nocturne de Job 4,12-21 (sur laquelle voir infra, § 7.4) ainsi que plusieurs passages où il est question de l'esprit dans le livre d'Osée.

27 Le thème du faux message est central dans 1 R 22 ainsi que dans d'autres textes prophétiques (notamment Isaïe et Osée) qui développent la polémique autour de la fausse prophétie, mais il n'est pas un thème portant dans d'autres contextes tels que le livre de Samuel ou celui des Juges. L'analyse de Hamori devrait donc être complétée par une approche qui prenne mieux en compte la diversité des contextes narratifs et historiques où les esprits apparaissent. 
où l'esprit prend la parole en tant que représentant générique de sa classe ${ }^{28}$. Enfin, Reed Carlson se fonde sur l'analyse des constructions verbales et syntaxiques employées lorsqu'il est question des rapports entre l'esprit et les êtres humains. Il considère qu'à chaque fois que l'esprit est le sujet d'une action et à chaque fois qu'un humain agit sous l'influence d'un esprit il est question d'un esprit de possession ${ }^{29}$. Par conséquent, il élargit considérablement la liste des esprits potentiellement conçus comme puissances agissantes, en distinguant deux typologies d'esprit: les « esprits de possession », qui affectent l'homme de manière transitoire et qui sont préposés à des tâches spécifiques, et les « esprits d'animation » (comme par exemple celui de Gen 1,2 et 2,7), qui possèdent les hommes de manière permanente.

Ces études proposent des solutions différentes au même problème. Étant donné que, comme nous le verrons par la suite, il n'y a pas de différence substantielle entre les modalités de fonctionnement de l'esprit de Yhwh et celui des autres esprits (les expressions employées sont les mêmes dans les deux cas), il faut alors définir des critères pour distinguer l'esprit comme faculté purement «psychologique », condition ou émotion humaine, et comme puissance externe, qu'elle soit d'origine divine ou autre. L'interaction entre esprits et êtres humains est compliquée non seulement car, comme nous l'avons vu, le langage des esprits est considéré comme particulièrement apte à exprimer certaines conditions humaines (certains « états d'esprit » serait-on tenté de dire), mais également en raison de la relation étroite exprimée par plusieurs textes bibliques entre rûah divin et rûah humain. Cette relation relève de l'idée, absolument centrale dans la Bible hébraïque, que Yhwh est la source de la vie pour les hommes et que son esprit est doté d'un pouvoir vivifiant ${ }^{30}$.

Même en adoptant une approche minimaliste, la proposition de Carlson me paraît fondée. Lorsque l'esprit n'est pas uniquement décrit comme l'envoi d'une substance «versée, répandue» par Yhwh, mais qu'il est lui-même le sujet actif d'un mouvement ou d'un acte qui semble impliquer une intentionnalité, il est effectivement doué d'une certaine agentivité. C'est le cas de plusieurs passages dans le livre des Juges, lorsque l'esprit revêt Gédéon $(l b s)^{31}$ ou qu'il trouble Samson $\left(p^{4} m \text {, « déranger », «troubler » }\right)^{32}$; le même vaut pour les

\footnotetext{
28 White 2014, p. 119-121.

29 Carlson 2019.

30 Outre le locus classicus de Gen 2,7 on peut voir, par exemple, Ps 51,12 et 104,29-3o ainsi que plusieurs passages en Isaïe $(32,15 ; 42,1 ; 44,3 ; 63,11)$ et la vision d'Ez 37 (voir notamment le verset 14).

31 Jug 6,34, mais on peut aussi comparer avec 1 Ch 12,19; 2 Ch 24,20.

32 Jug 13,25 .
} 
actions décrites en 1 Samuel où l'esprit prophétique « entre par la force » $(s ̦ l h)$ en Saül, le «terrorise» $\left(b^{\prime} t\right)$, et enfin se retire $\left(s^{\prime} h\right)^{33}$.

Quelques questions demeurent cependant. On se demande en effet dans quelle mesure on ne resterait pas uniquement dans l'ordre du langage, à savoir de la métaphore. L'esprit mauvais, l'esprit de sagesse, de jalousie, de grâce et de supplication, d'impureté, de prostitution, de torpeur, de jugement etc., que Yhwh envoie sur, ou verse dans, les êtres humains ainsi que l'esprit de prophétie dont il remplit ses prophètes devraient-ils être interprétés comme de simples descriptions métaphoriques d'un état émotif ou psychologique ou d'une «inspiration » divine générique ? Ou impliquent-ils, dans tous les cas, l'action d'une puissance agissante, comme c'est le cas de l'esprit de mensonge qui prend la parole en $1 \mathrm{R} 22$ et comme Carlson semble le suggérer? Nous ne pouvons certainement pas répondre à ces questions de manière univoque, notamment car il faut tenir compte de la diachronie des textes bibliques, quasiment ignorée, d'ailleurs, par les études mentionnées plus haut. Comme le souligne Mareike Verène Blischke ${ }^{34}$, la notion d'esprit fait déjà l'objet de plusieurs développements à l'intérieur de la Bible hébraïque. Alors que certaines différences sont à expliquer par des compréhensions de l'esprit propres à chaque corpus (on pourrait comparer à cet égard Isaïe avec Ézéchiel), d’autres sont la conséquence de dépendances littéraires directes (par exemple du livre des Juges par rapport aux livres de Samuel) ; d'autres encore relèvent de changements plus généraux concernant la conceptualisation de rûaḥ, qui semble acquérir de l'importance à l'époque postexilique. Cette importance est notamment confirmée dans les traditions sapientielles par le rôle de premier rang attribué à l'esprit dans la création ; rôle qui atteint son apogée dans le livre de la Sagesse $^{35}$. Il devient alors d'autant plus intéressant de comprendre la manière dont la LXX s'inscrit dans ces processus.

Un certain nombre de remarques sur le langage employé dans les différents contextes mentionnant des esprits permet de formuler quelques hypothèses de recherche. Premièrement, il faut observer que, lorsque rûah décrit une condition interne à l'individu, l'on n'utilise pas l'expression rûah suivie par un substantif de spécification qui correspond à la manière la plus fréquente d'exprimer

\footnotetext{
33 Voir infra, p. $245^{-248 .}$

34 Blischke 2019, voir surtout p. 289-298.

35 Sur l'importance de l'esprit à l'époque postexilique, voir également MacDonald 2013. Blischke (2019, p. 63-75) considère également comme secondaires une partie des références à l'esprit de Yhwh dans le Pentateuque, qui visent à insérer le motif de l'inspiration divine dans des passages clés, tels que le début (la création en Gen 1,2); le désert (la construction de la tente de la rencontre en $\operatorname{Ex} 28,3 ; 31,3 ; 35,31)$ et la fin, avant l'entrée dans la terre promise (la descente de l'esprit sur Josué en Deut 34,9).
} 
la fonction adjectivale en hébreu (voir infra, tableau 9), mais l'on préfère avoir recours à un adjectif (ou à un substantif) suivi et délimité par rûaḥ en tant que complément de limitation qui indique le domaine d'action de l'adjectif (voir tableau 6 ci-dessous). C'est le cas des expressions «opprimé dans l'esprit» (1 S 1,15); «court/grand d'esprit» (Prov 14,29); «errant d'esprit» (Is 29,24), «accablé d'esprit» (Is 54,6) et d'autres encore. Ces occurrences se rencontrent davantage, mais pas exclusivement, dans la littérature sapientielle et elles sont fréquentes dans le livre des Proverbes qui a pour objet une réflexion sur la nature humaine. Comme le montre le tableau 6, dans la LXx, et cela dès le Pentateuque, ces passages sont, pour la plupart, rendus par des expressions qui ne traduisent pas littéralement rûah par $\pi v \varepsilon \hat{\mu} \alpha^{36}$. Cette démarche confirme que l'hébreu biblique connaît l'usage de rûah dans un sens purement psychologique, n'impliquant aucune référence à des puissances externes, et que cet usage était encore compris par la plupart des traducteurs :

TABLEAU 6 Rûah et fonctions psychiques

ותהיין מרת רוח ליצחק ולרבקה Gen 26,35 amertume d'esprit

Ex 6,9 מקצר רוח

(«court») faible d'esprit

וקצר רוח 14,29 Prov

קשת רוח 1,15

opprimé dans l'esprit

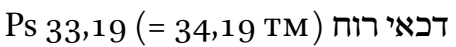

dévasté, contrit dans l'esprit

שבר ברוח 15,4 ש

rupture d'esprit

גבה רוח 16,18

(« exalté») superbe d'esprit

Prov 16,19 שפל רוח

humble d'esprit

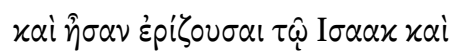

$\tau \hat{\eta} \mathrm{P} \varepsilon \beta \varepsilon \kappa \kappa \alpha$

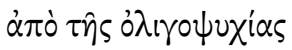

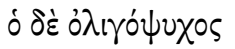

$(\hat{\eta} \sigma x \lambda \eta \rho \dot{\eta} \dot{\eta} \mu \dot{\varepsilon} \rho \alpha)$

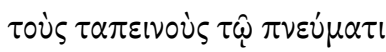

$\pi \lambda \eta \sigma \theta \dot{\eta} \sigma \varepsilon \tau \alpha \iota ~ \pi \nu \varepsilon \dot{\mu} \mu \alpha \tau \sigma \varsigma$

какочробо́n一

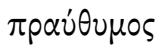

36 Comme le montrent le cas du Ps 33,19, Qoh 7,8, et Is 29,24 il s'agit d'une tendance générale, et non d'une règle stricte. En Ps 33,19, le traducteur choisit de rendre rûah par $\pi v \varepsilon \hat{u} \mu \alpha$ car il veut probablement préserver le parallélisme structurel avec le « cœur » de l'hémis-

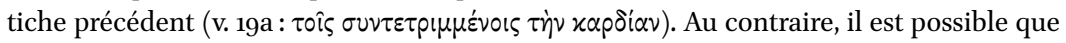
le traducteur de Qohéleth ait cherché à produire un effet de variatio en 7,8. Le traducteur des Proverbes évite rigoureusement l'usage de $\pi v \varepsilon \hat{u} \mu \alpha$ au sens psychique. En Prov 15,4

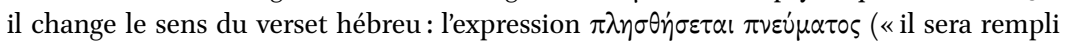
d'esprit ») dans le texte grec fait référence à l'inspiration divine. 
TABLEAU 6 Rûah et fonctions psychiques (cont.)

Prov $17,27^{a}$ קר רוח

froid/noble d'esprit

ארד רוח מגבה רוח

(mieux) quelqu'un «long » d'esprit (patient)

que quelqu'un « exalté » d'esprit (superbe)

תעי רוח

errant d'esprit

Is ע4,6 עצובת רוח

accablé d'esprit

Is 66,2 נכה רוח

frappé dans l'esprit

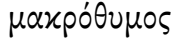

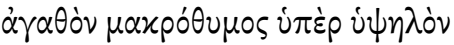

$\pi v \varepsilon \dot{\mu} \mu \alpha \tau$

oi $\tau \hat{\omega} \pi v \varepsilon \dot{\mu} \mu \alpha \tau ı$ I $\pi \alpha \nu \omega^{\prime} \mu \varepsilon v 0$ เ

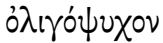

ทंбóx10v

a Forme ketiv : wqr, «froid »; forme qere :yqr, «précieux »

Deuxièmement, l'inspiration divine n'est pas toujours décrite dans les mêmes termes. On peut comparer, à cet égard, la formulation des Juges avec celle des Chroniques. En Jug 9,23 Yhwh «envoie un esprit mauvais parmi Abimelech et parmi les Sichémites» (וישלח אלהים רוח רעה בין אבימלך ובין בעלי שכם). La formulation qui décrit l'hostilité entre Abimelech et les habitants de Sichem est assez singulière : on mentionne l'envoi d'un esprit qui reçoit le qualificatif de $r \bar{a} \hat{a}$, mauvais. Cette formulation est, à bien des égards, différente de ce que l'on trouve, par exemple, en $2 \mathrm{Ch} 21,16$ où il est encore question d'hostilité réciproque. Dans ce deuxième cas, on dit simplement que «Yhwh suscita contre

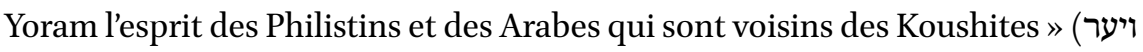
(יהוה על יהורם את רוח הפלשתים והערבים אשר על יד כושים. Lesprit, traduit parfois par «colère » (mais il serait préférable de le traduire par « une attitude hostile à »), semble indiquer l'intériorité de l'individu ; cet usage est également attesté dans d'autres textes bibliques ${ }^{37}$. En ce sens, la formule «Yhwh suscita/réveilla l'esprit de tel ou tel», qui se retrouve en 1 Ch 5,26 (esprit des rois d'Assyrie), ${ }_{2}$ Ch 36,22, Esdr 1,1 (esprit de Cyrus) et 1,5 (esprit des habitants de Juda) ainsi qu'en Agg 1,14 (esprit de Zorobabel et du grand prêtre Josué), semble être une métaphore pour décrire l'inspiration divine qui préside à la construction du Second Temple, alors que l'esprit mauvais dans les Juges fonctionne comme une véritable puissance envoyée par Yhwh, une rûah rāâa proche de celle qui est décrite en $1 \mathrm{~S}$ 16. Il demeure cependant difficile de retracer un parcours chronologique qui amènerait d'une ancienne externalisation de l'esprit à sa

37 Voir, par exemple, «mon esprit » en Ps 32,2; Is 26,9 ; et en grec Bar 3,1; Dan 3,39. 
progressive intériorisation ${ }^{38}$. La formulation de Jug 9,23 est en accord avec la tendance à représenter les actions de l'esprit de Yhwh comme une puissance agissante dans le reste du livre. Une autre expression à considérer est «je mettrai en lui un esprit ... », en 2 R 19,7, qui décrit d'une manière encore différente l'inspiration divine. Yhwh, en parlant par la bouche du prophète Isaïe, annonce au roi Ézéchias qu'il est sur le point de pousser le roi d'Assyrie Sennachérib à se retirer à Ninive, suite à la nouvelle d'une attaque contre ses troupes par le roi des Nubiens. Il ajoute, en outre, qu'il sera tué, une fois rentré chez lui ${ }^{39}$ :

הנני נתן בו רוח ושמע שמועה ושב לארצו והפלתיו בחרב בארצו

Et je mettrai en lui un esprit tel qu'il entendra une nouvelle et il rentrera dans sa terre, et je le ferai tomber par l'épée dans sa terre.

La formulation est ambiguë : elle peut faire référence autant à un concept générique d'inspiration qu'à une entité agissante. Elle n'est pas sans rappeler le $\delta \alpha i ́ \mu \omega \nu$ que décrit Plutarque, qui avait inspiré à l'athénien Démade la mauvaise idée de partir en Macédoine où il fut tué à son arrivée ${ }^{40}$. Il vaut, en outre, la peine de remarquer que le verbe $n t n$ est souvent employé en connexion avec rûah dans la Bible hébraïque ${ }^{41}$. Il apparaît, notamment, en contexte prophé-

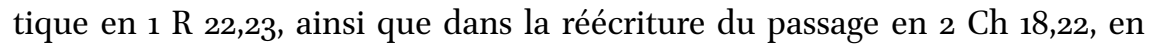
relation à l'esprit de mensonge. La proximité de ces formulations est assez frappante.

Dernièrement, il faut signaler le cas particulier du livre des Nombres, au chapitre 5 , versets 14 et 30 , dans un contexte légal où il est question d'un esprit de jalousie qui saisit un homme et des actions qu'il est nécessaire d'entreprendre pour prouver l'innocence de la femme :

38 Alors que pour Chroniques et Esdras une datation à l'époque hellénistique est parfois envisagée, pour Aggée la situation est différente. La plupart des chercheurs reconnaît le caractère secondaire du cadre narratif, qui inclut Agg 1,14, par rapport à l'oracle originaire, mais il est difficile de pouvoir situer le cadre beaucoup plus tard qu'au début de l'époque perse (Wöhrle 2006, p. 384-385; Leuenberger 2015, p. 103; id. 2020, p. 230-231; Rückl, 2021, p. 59-65). Pour un modèle qui prévoit une fragmentation supplémentaire du cadre au niveau rédactionnel, voir Hallaschka 2011, p. 134-135. Blischke (2019, p. 257-258) avance l'hypothèse d'un lien rédactionnel entre Agg 1,14; Esdr 1,1, 5 ; 2 Ch 36,22.

39 La prédiction divine se réalise ponctuellement en $2 \mathrm{R}$ 19,35-37. Le même récit apparaît également en Is 37,7 et $36-38$.

40 Plut., Phoc. 3 o, 9 et supra, p. 70.

41 Num 11,25 et 29 ; Neh 9,20; Is 42,1, 5 ; Ez 11,19;13,26;36,26-27; 37,6; 37,14. En Qoh 12,7 rûah indique clairement le souffle. 
ועבר עליו רוח קנאה וקנא את אשתו והוא נטמאה או עבר עליו רוח קנאה וקנא את

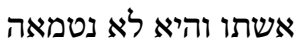

S'il passe sur lui un esprit de jalousie et il devient jaloux de sa femme, et elle se soit contaminée, ou s'il passe sur lui un esprit de jalousie et il devient jaloux de sa femme, et elle ne se soit pas contaminée ...

Cette formulation se différencie des autres mentions de rûah dans la Bible hébraïque. Tout d'abord, il s'agit d'un rare cas où l'origine de l'esprit n'est pas spécifiée : rien ne laisse entendre qu'il soit envoyé par Yhwh, mais il ne semble pas non plus résider de manière permanente dans l'individu. En outre, l'expression ' $b r$ 'l, «passer sur», n'est jamais employée ailleurs pour décrire le mouvement des esprits. De plus, cette loi répond à une situation de jalousie injustifiée en couvrant un cas de figure qui n'est pas nécessairement motivé par une mauvaise conduite de la femme, mais qui n'est pas discuté par les autres lois sur l'adultère ${ }^{42}$. Enfin, le cas est résolu par ordalie, en remettant la décision concernant la culpabilité de la femme à la volonté divine. Ces éléments indiquent que nous ne sommes pas ici simplement dans le cadre du langage métaphorique, ni d'une vision psychologisante des esprits, mais que nous avons affaire à une puissance, l'esprit de jalousie, qui s'empare du mari de manière incontrôlable, sans qu'il y ait une motivation dans le comportement de l'épouse.

Ces considérations d'ordre linguistique appuient donc la proposition de Hamori qui est d'inclure au moins Jug 9,23 ainsi que 2 R 19,7 parmi les textes qui décrivent les esprits comme puissances en action. Ces mêmes considérations suggèrent en outre que le texte de Nombres 5 décrit un esprit d'origine inconnue, mais dont l'agentivité ne se différencie pas de celle des esprits divins ${ }^{43}$. Au contraire, avec l'esprit de jalousie nous sommes tout à fait au cœur de la problématique liée à la possession par les esprits, ainsi qu'à leur contrôle de la part de Yhwh.

\subsection{Esprits divins et possession}

Avec la possible exception de l'esprit en Nombre 5 que je viens de discuter et d'un cas dans le livre de Job que j'analyserai plus tard ${ }^{44}$, tous les esprits dans

42 On peut comparer Lev 22 et Deut 20.

43 Esther Hamori (2010, p. 18) exclut en revanche l'esprit de jalousie de son dossier car il ne répond pas aux critères narratologiques qu'elle adopte qui demeurent, toutefois, à mon avis, insuffisants.

44 Voir infra, § 7.4. 
la Bible hébraïque sont des puissances subordonnées à Yhwh. Le problème du rapport entre Yhwh et ses esprits émerge clairement dans la scène d'assemblée céleste en 1 R 22,19-23, notamment lorsque l'on compare la version de la LXX avec celle du TM. C'est donc sur cet épisode que je vais d'abord focaliser mon attention.

Depuis l'étude d'Ernst Würthwein ${ }^{45}$, les chercheurs s'accordent sur le fait que $1 \mathrm{R} 22$ ne constitue pas une unité littéraire. Plus précisément, le récit concernant le prophète Michée et sa vision du trône céleste n'appartient pas au noyau primitif de la chronique royale, mais a été inséré à un stade ultérieur ${ }^{46}$. L'épisode est raconté également en 2 Ch 18,18-22, mais ce passage ne contient pas de variantes significatives quant à la représentation des esprits, ni en grec ni en hébreu. Je vais donc me concentrer sur le texte de $1 \mathrm{R} 22$. Ici, la vision de Michée s'inscrit dans le contexte plus large du débat autour de la vraie et de la fausse prophétie. Le chapitre commence avec une visite du roi de Juda, Josaphat, auprès du roi d'Israël Achab. Josaphat propose à Achab d'attaquer le royaume voisin d'Aram. Lors d'une consultation publique prise avant la bataille pour connaître le sort du conflit, les deux souverains siègent chacun sur son trône devant quatre-cents prophètes de Samarie en extase qui leur annoncent la victoire. Le roi de Juda demande qu'un prophète de Yhwh soit également consulté: Michée est convoqué et il relate devant tous sa vision d'une assemblée céleste présidée par Yhwh, au cours de laquelle un esprit s'offre volontaire pour tromper les prophètes du roi Achab et être un esprit mensonger sur leurs bouches. Il révèle ainsi la nature trompeuse de l'oracle rendu par les quatre cents prophètes, en suscitant leur colère et celle du roi (1 $\mathrm{R} 22,24-28)$. La conclusion du récit, qui voit la mort d'Achab tué lors d'une bataille malgré ses tentatives de camouflage, confirme la vérité des paroles du prophète de Yhwh.

45 Würthwein 1967.

46 En revanche, plusieurs propositions ont été avancées quant au modèle rédactionnel et quant à l'origine du noyau primitif. Würthwein considère les versets 19 à 23 comme dérivant d'une réflexion tardive sur le mauvais usage de l'esprit de la part des prophètes; d'après Helga Weippert (1988), qui développe un modèle assez complexe, l'épisode est composé de deux sources distinctes, une qui racontait la mort héroïque d'un roi israélite et l'autre qui contenait une prophétie sur sa victoire. L'intégration de ces sources dans le contexte des guerres entre Israël et Aram aurait amené à une série de remaniements successifs. Hedwige Rouillard (1993), et plus récemment Steven McKenzie (2019, p. 207213) ainsi que Knauf (2019, p. 482-486) estiment qu'une rédaction «prophétique » soit responsable de l'insertion de tout le récit concernant Michée (versets $5^{-28}$ ), laquelle d'après Knauf aurait subi encore quelques modifications à l'époque hasmonéenne. Cette hypothèse demeure, à mon avis, la plus vraisemblable car, du point de vue narratif, les parallélismes entre la scène terrestre (versets $5^{-17}$ ) et la scène céleste (versets $18-23$ ) sont évidents et les deux scènes semblent bien intégrées à la conclusion du récit prophétique (versets 24-28). 
La vision du conseil divin présente quelques différences importantes dans les traditions grecques qui nous ont été transmises et qui méritent d'être regardées de près (voir tableau 7 ):

TABLEAU $7 \quad 1$ Rois $22,19-23^{a}$

TM

19 ויאמר לכן שמע דבר

יהוה ראיתי את יהוה

ישב על כסאו וכל צבא יהוח ראיתי יהות

השמים עמד עליו מימינו

ומשמאלו

20 ויאמר יהוה מי יפתה

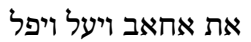

ברמת גלעד ויאמר זה

בכה וזה אמר בכה

21 ויצא הרוח ויעמד לפני יהוה ויאמר אני אפתנו

ויאמר יהוה אליו במה ויאמר אני פתנו

22 ויאמר אצא והייתי

רוח שקר בפי כל נביאיו

ויאמר תפתה וגם תוכל

צא ועשה כן
$\operatorname{LXX}(\mathrm{B})$

19 x

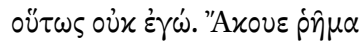

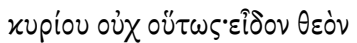

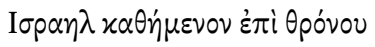

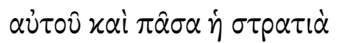

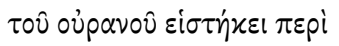

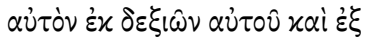

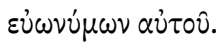

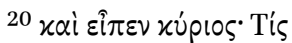

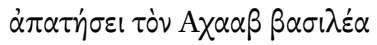

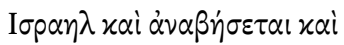
$\pi \varepsilon \sigma \varepsilon \hat{\imath} \tau \alpha$ เ हे $\mathrm{P} \varepsilon \mu \mu \alpha \theta \Gamma \alpha \lambda \alpha \alpha \delta$;

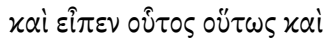

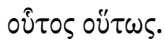

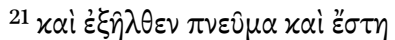

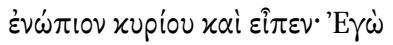
$\dot{\alpha} \pi \alpha \tau \dot{\eta} \sigma \omega \alpha$ ứv.

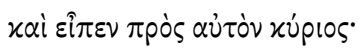

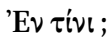

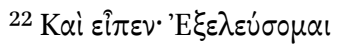

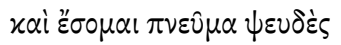

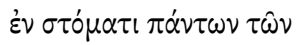

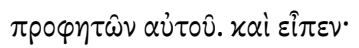
'A $\pi \alpha \tau \dot{\eta} \sigma \varepsilon เ \varsigma$

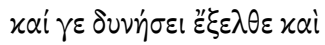

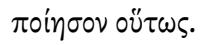

LXX (L)

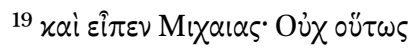

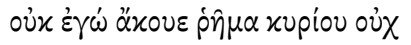

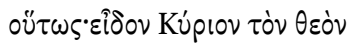

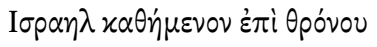
$\alpha \hat{\tau} \tau 0 \hat{x} x \alpha i$ $\pi \hat{\alpha} \sigma \alpha \dot{\eta} \sigma \tau \rho \alpha \tau i \dot{\alpha} \tau 0 \hat{v}$

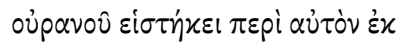

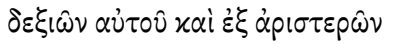

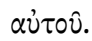

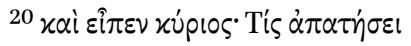

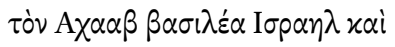

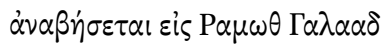

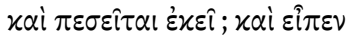

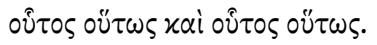

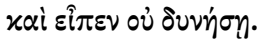

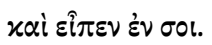

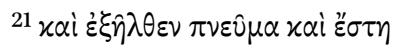
घे

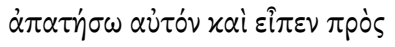

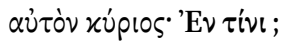

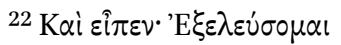

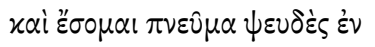
$\sigma \tau \dot{0} \mu \alpha \tau \iota \pi \dot{\alpha} \nu \tau \omega \nu \tau \hat{\omega} \nu \pi \rho \circ \varphi \eta \tau \hat{\omega} \nu$

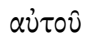

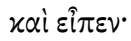

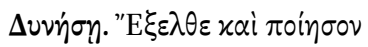

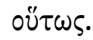

a Dans les colonnes 2 et 3 , le texte B a été reproduit d'après la version de Brooke, McLean et Thackeray 1930 (1 Rois) et 1927 (1 Samuel); le texte L suit l'édition de Fernández Marcos et Busto Saiz 1992 (1 Rois) et 1989 (1 Samuel). Le texte L est transmis par les mss. no. 19, 82, 93, 108, 127 dans l'édition en préparation pour la série Septuaginta Unternhemen de Göttingen par Julio Trebolle et Pablo Torijcano ( $=$ boc $_{2} \mathrm{e}_{2}$ dans Brooke, McLean et Thackeray 1930), ainsi que par des citations des pères de l'Église. Ces manuscrits représentent une famille homogène pour tous les livres historiques. 


$\begin{array}{lll}\text { TM } & \text { LXX (B) } & \text { LXX (L) }\end{array}$

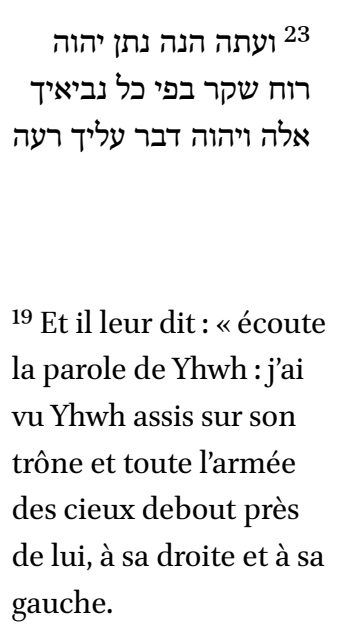

${ }^{20}$ Alors Yhwh dit : "Qui persuadera Achab pour qu'il monte et tombe à Ramat Gilead ?" et l'un disait une chose, l'autre une autre chose.

${ }^{21}$ Alors cet esprit sortit et se présenta devant Yhwh, puis il dit: "Je le persuaderai”. Et Yhwh lui dit: "Comment?". 22 et il répondit : "Je sortirai et je serai un esprit mensonger sur la bouche de tous ses prophètes ". Et (Yhwh) dit: "Tu le persuaderas, effectivement tuen auras le pouvoir. Va, et agis ainsi".

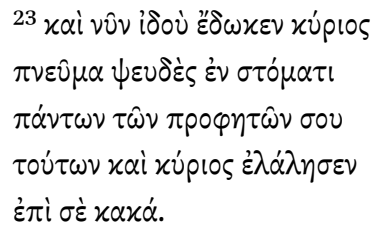

${ }^{19}$ Et Michaias dit: « Pas ainsi, pas moi. Écoute la parole de Yhwh. Pas ainsi. J'ai vu le dieu d'Israël assis sur son trône, et toute l'armée des cieux se tenait autour de lui, à sa droite et à sa gauche,

20 et le Seigneur dit : "Qui trompera Achab, roi d'Israël, pour qu'il monte et tombe à Remat Galaad ?" Et l'un dit une chose, l'autre une autre chose.

${ }^{21}$ Alors un esprit sortit et se tint devant le Seigneur et dit : "Moi je le tromperai". Et le Seigneur lui dit :

"Comment?".

22 et il répondit : "Je sortirai et je serai un esprit mensonger dans la bouche de tous ses prophètes". Et (Yhwh) dit : "Tu le tromperas, effectivement tu en auras le pouvoir. Va, et agis ainsi".

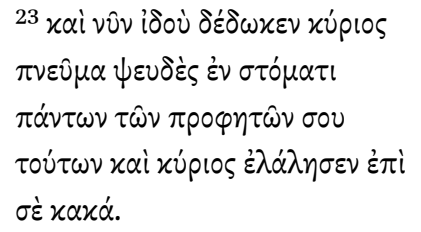

${ }^{19}$ Et Michaias dit: « Pas ainsi, pas moi. Écoute la parole de Yhwh. Pas ainsi. J'ai vu le Seigneur dieu d'Israël assis sur son trône, et toute l'armée des cieux se tenait autour de lui, à sa droite et à sa gauche, 20 et le Seigneur dit: "Qui trompera Achab, roi d'Israël, pour qu'il monte à Remat Galaad et tombe là-bas ?" Et l'un dit une chose, l'autre une autre chose. Et il dit: “Tu n'en auras pas le pouvoir". Et il répondit : "par toi".

${ }^{21}$ Alors un esprit sortit et se tint devant le Seigneur et dit : "Moi je le tromperai". Et le Seigneur lui dit : "Comment?". 22 et il répondit : "Je sortirai et je serai un esprit mensonger dans la bouche de tous ses prophètes". Et (Yhwh) dit : "Tu en auras le pouvoir. Va, et agis ainsi". 
TABLEAU $7 \quad 1$ Rois 22,19-23 (cont.)

$\begin{array}{lll}\text { TM } & \text { LXX (B) } & \text { LXX (L) }\end{array}$

$\begin{array}{ll}{ }^{23} \text { Et maintenant voici } & { }^{23} \text { Et maintenant voici } \\ \text { que Yhwh a mis un } & \text { que Yhwh a mis un esprit } \\ \text { esprit mensonger sur } & \text { mensonger dans la bouche } \\ \text { la bouche de tous ces } & \text { de tous tes prophètes } \\ \text { prophètes à toi, c'est } & \text { que voici, c'est Yhwh qui } \\ \text { Yhwh qui a prononcé le } & \text { a prononcé les malheurs } \\ \text { mal contre toi ». } & \text { contre toi ». }\end{array}$
${ }^{23}$ Et maintenant voici que Yhwh a mis un esprit mensonger dans la bouche de tous tes prophètes que voici, c'est Yhwh qui a prononcé les malheurs contre toi ».

Tout d'abord quelques remarques doivent porter sur le texte hébreu. Comme nous l'avons déjà observé, l'assemblée divine qui se tient autour de Yhwh crée un effet de miroir assez ironique par rapport à l'assemblée terrestre réunie à Samarie pour la consultation prophétique, en mettant en parallèle les deux trônes des rois avec le trône de Yhwh et, par conséquent, les prophètes avec les esprits ${ }^{47}$. Il faut toutefois noter que la scénographie du conseil divin présente une structure plus articulée que l'assemblée qui se réunit autour d'Achab et Josaphat. Elle est hiérarchiquement structurée sur plusieurs niveaux, dans lesquels chaque position reflète le statut des participants. L'armée des cieux, vraisemblablement composée des corps célestes, occupe les postes autour de Yhwh, en se tenant debout (' $m d$ ) à la droite et à la gauche du trône et en fonctionnant comme une garde rapprochée du dieu (verset 19) ${ }^{48}$. Le fait que l'esprit qui s'annonce volontaire sorte et s'avance pour se présenter devant Yhwh fait, en revanche, penser que les esprits occupent une place plus éloignée du trône, correspondant à une position de second rang (versets 20-21). La présence de l'article devant rûaḥ n'indique pas, comme on l'a parfois suggéré, qu'il s'agit du seul esprit présent, mais qu'il est l'un des esprits de l'assemblée («celui-ci »); cela implique en outre que, comme l'a démontré White, rûaḥ dénote ici un titre, une fonction à l'intérieur de la cour céleste, à l'instar de haśśāțān en Zach 3 et Job $1-2^{49}$. Les verbes utilisés pour décrire le mouvement de l'esprit, qui doit sortir de son rang pour se tenir devant le trône de Yhwh (wayyēṣe hārûaḥ wayya ămōd, «il sortit et se présenta/se tint debout»), laissent entrevoir une étiquette de cour qui fonctionne par des règles précises. Au verset 22,

47 Par exemple Rouillard 1993, p. 104; McKenzie 2019, p. 205.

48 Comme d'ailleurs dans les autres scènes de conseil divin en Is 6 et Job 1-2.

49 White 2014, p. 119-121. Voir infra, p. 282-283. 
l'expression wagam tûkăl, « effectivement tu en seras capable», «tu en auras le pouvoir », exprime l'autorisation divine accordée à la mission et annonce son succès, en mettant fin au débat en cours parmi la multitude des participants à l'assemblée. Une telle compréhension de la structure du conseil confirme la faiblesse des interprétations qui font de cet esprit une émanation ou une personnification de Yhwh lui-même ${ }^{50}$. La présentation de l'assemblée divine met donc l'accent sur ses aspects hiérarchiques ainsi que sur sa procédure strictement codifiée qui recoupe, au moins en partie, le déroulement des assemblées royales au Proche-Orient ancien, et au Levant plus spécifiquement ${ }^{51}$.

Une série d'éléments proviennent des versions grecques. À cet égard, il faut tenir compte des différences entre la version préservée par le Vaticanus (B) et le texte dit «antiochien» ou «lucianique» (L). Les chercheurs estiment que pour cette section des livres grecs des Règnes, $\mathrm{B}$ relève de la révision kaige, un ensemble de corrections du grec ancien faites sur la base d'un texte hébreu proto-massorétique autour du $\mathrm{I}^{\mathrm{er}} \mathrm{s}$. av. notre ère qui est, peut-être, à mettre en relation avec l'activité de Théodotion ${ }^{52}$. En revanche le texte L, même s'il contient des améliorations stylistiques secondaires (par exemple $\alpha$ pı $\sigma \tau \varepsilon \rho \hat{\nu}$ au lieu de $\varepsilon \dot{\omega} \omega \nu v ́ \mu \omega \nu$ pour indiquer la gauche au verset 19), ainsi que des interpolations hexaplaires, préserve également des éléments anciens (voir par exemple

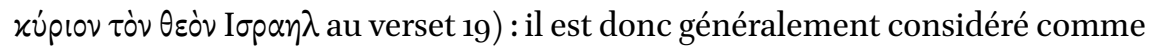
un bon témoin de la Septante ancienne ${ }^{53}$. Le texte L montre plusieurs divergences par rapport à l'hébreu : on ne discutera ici que de celles qui concernent le traitement de l'esprit. En L, l'interaction entre l'esprit et la divinité est plus développée que dans le texte $\mathrm{B}$ et dans le TM, et Alfred Rahlfs reconnaissait déjà que ce passage était d'origine prélucianique ${ }^{54}$. Pourtant le verset 20 dans

50 À cet égard, dans la conclusion de la scène (verset 24), l'un des prophètes de la cour du roi Achab se met en colère et frappe Michée en disant: « Par où l'esprit de Yhwh est sorti de moi pour parler à toi ? ». Ce ne sont donc que les faux prophètes qui ne sont pas capables de distinguer l'esprit de Yhwh de l'esprit de mensonge.

51 Sur les aspects bureaucratiques de l'assemblée divine au Levant, on peut voir Mullen 1980 et M. Smith 2001, p. 41-53. On peut également comparer les audiences de Salomon avec Bethsabée et Nathan en 1 R 1,15-28. La comparaison entre étiquette royale et étiquette divine mériterait un travail de détail qui, à ma connaissance, n’a pas encore été fait. Pour une introduction aux audiences royales au Proche Orient ancien, on peut voir Garelli 1974, Joannès 2007, Radner 2011, Pruß 2019.

$5^{2}$ D'après Barthelemy (1963) les corrections kaige dans les Règnes couvrent les sections 2 Regn 11,2-3 Regn 2,11 et 3 Regn 22-4 Regn.

53 Les rapports entre $\mathrm{B}, \mathrm{L}$, les corrections kaige et la Septante ancienne sont très complexes. Pour un bilan de recherche détaillé, voir Hugo 2006; voir également les remarques méthodologiques de Law et Kauhanen 2010.

Rahlfs 1965, I, p. 96-98; III, p. 646. 
le texte L n'a pas vraiment de sens : on comprend mal l'articulation entre l'ex-

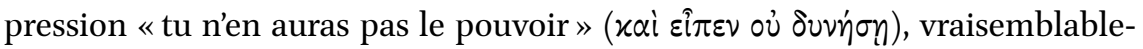
ment attribuée à Yhwh, avec la réponse «en/par toi » ( $\left.\varepsilon^{2} v \circ \mathrm{\sigma}\right)$.

Dans un article récent, Julio Trebolle ${ }^{55}$ suggère le déplacement d'une partie

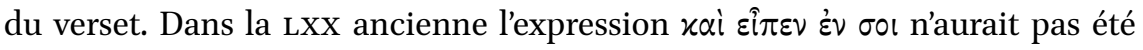
à la fin du verset 20 , mais à la fin du verset 21, ce qui donnerait la restitution suivante:

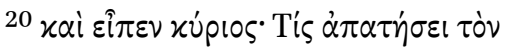

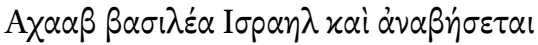

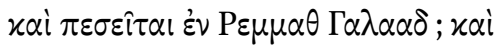

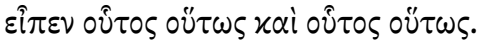

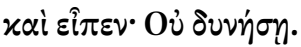

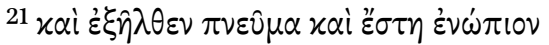

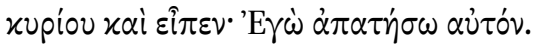

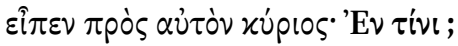

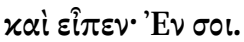

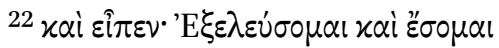

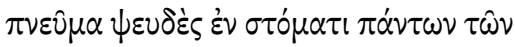

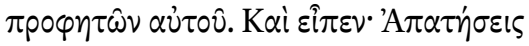

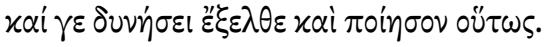

${ }^{20}$ Yhwh dit : «Qui va tromper Achab roi d'Israël, pour qu'il monte à Ramaat de Galaad et qu'il y meure? ». L'un disait une chose, l'autre une autre. Et (Yhwh) dit: tu n'auras pas le pouvoir.

${ }^{21}$ Alors un esprit sorti et se tint devant Yhwh, et il dit: «Moi je le tromperai ». Et le Seigneur lui a dit : «Comment/ par qui ?» et il répondit: «par toi ». ${ }^{22}$ Et il (l'esprit) dit: « Je sortirai, et je serai un esprit mensonger dans la bouche de tous ses prophètes ». Et il (Yhwh) dit : « Tu le persuaderas, vraiment tu en auras le pouvoir. Sors et agis de cette manière ».

D'après Trebolle, le texte $\mathrm{L}$ avec ce petit déplacement correspondrait entièrement à la LXx ancienne et relèverait d'une Vorlage hébraïque différente du $\mathrm{TM}$, qu'il reconstruit à partir du grec. Il estime que ce passage aurait ensuite été effacé pour des raisons théologiques, à cause d'une interaction trop développée entre la divinité et les membres de la cour ou à cause du fait que l'association de Yhwh avec le mensonge était perçue comme problématique. Quelques problèmes demeurent cependant avec cette reconstruction.

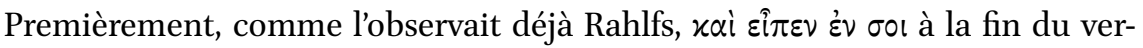
set 20 - à savoir exactement dans la position où il a été transmis dans la tradition manuscrite - pourrait traduire également ' $m r b k h$ (dans ce cas, bəkô, « ainsi », aurait été lu comme bəkâ, « en/par toi », à l'instar, par exemple, de $1 \mathrm{R}$ 
$10,9)^{56}$. Deuxièmement, la reconstruction de Trebolle n'est pas toujours entièrement justifiée à la lumière du texte grec: il n'est pas nécessaire d'envisager un hébreu bəmî («par qui ? ») au lieu de bammâ («comment?») comme correspondance de $\dot{\varepsilon} \nu \tau i v$ c car le pronom interrogatif en grec peut être à la fois masculin ou neutre ${ }^{57}$. Troisièmement, la différence de contenu entre TM et L ne me paraît pas si significative. L'origine divine du pouvoir de l'esprit est de facto sanctionnée déjà dans le TM. Elle est annoncée au verset 22 («Tu le persuaderas, effectivement tu en auras le pouvoir. Va, et agis ainsi ») et réaffirmée encore au verset 23 par Michée : «Et maintenant voici que Yhwh a mis un esprit mensonger dans la bouche de tous tes prophètes que voici, c'est Yhwh qui a prononcé les malheurs contre toi ». Par conséquent, les motivations théologiques qui, d'après Trebolle, auraient pu être à l'origine de la suppression de ce passage en hébreu ne me paraissent pas très conséquentes. En revanche, le supplément du texte L pourrait bien s'expliquer comme l'exégèse d'un passage assez dense qui essaie d'articuler plus clairement la dynamique de question et réponse entre Yhwh et l'esprit, laquelle demeure implicite en hébreu, comme le remarque d'ailleurs très justement Trebolle ${ }^{58}$. Il est, certes, possible que ce développement soit antérieur à la version lucianique, mais il est difficile de savoir s'il remonte effectivement à une Vorlage hébraïque ou s'il s'agit d'une évolution interne au grec. Il est également possible que l'on ne puisse plus reconstruire entièrement le texte de la LXX ancienne pour ce passage.

Une autre différence remarquée par les chercheurs est l'absence de l'article en grec devant $\pi v \varepsilon \hat{\mu} \mu \alpha$ au verset 21. Comme le suggère Knauf, une telle absence pourrait indiquer que le traducteur n'a pas compris hārûah comme le titre désignant un fonctionnaire de l'assemblée, mais comme une entité individuelle, un des nombreux esprits qui entouraient la cour céleste ${ }^{59}$. Par conséquent, l'élimination de l'article relève d'une adaptation stylistique, vu que rûah est nommé ici pour la première fois. Comme nous le verrons par la suite, cette interprétation est en accord avec l'évolution plus générale de la représentation des esprits dans la LXX.

$56 \quad$ Rahlfs 1965 , III, p. 646.

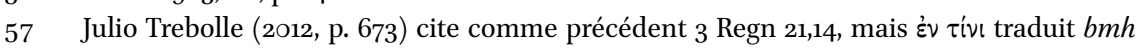
de manière systématique en 1 Regn 6,$2 ; 14,38 ; 29,4$ et 2 Regn 21,3. De manière générale, la reconstruction de Trebolle est faite à la lumière d'une évaluation très optimiste du statut de la Vieille Latine comme témoin du texte proto-massorétique. Toutefois, les sources disponibles pour la reconstruction de la Vieille Latine sont très fragmentaires et aucune variante significative n'est attestée par rapport au TM dans les versets qui sont examinés ici. Une certaine prudence est donc recommandée : voir, à cet égard, les remarques de Fernández Marcos 1994, p. 71-87, et Kauhanen 2018.

$5^{8} \quad$ Trebolle 2012, p. 672.

$59 \quad$ Knauf 2019, p. 5 ०5. 
Néanmoins, l'un des apports les plus importants de ce récit consiste en l'établissement d'une relation étroite entre possession par l'esprit et activité prophétique. On retrouve ce lien dans le cycle d'Elie et Élisée dans les livres des Rois : l'épisode montre la manière dont l'esprit peut être transmis d'un prophète à l'autre. Le lien entre esprit et prophétie a, en outre, un précédent narratif important dans le Pentateuque, lorsque les soixante-dix anciens reçoivent par Yhwh une partie de l'esprit de Moïse et commencent à prophétiser ${ }^{60}$. Mais le contexte où le lien entre esprit et prophétie est thématisé avec le plus de détails est sans doute l'histoire de Saül et David dans le premier livre de Samuel. En 1 S 16,14-23, le jeune David, appelé au service du roi Saül, le soulage du tourment provoqué par un mauvais esprit en jouant un instrument à corde ; 1 S 18,10-11 relate l'attentat manqué de Saül contre David, lorsque ce dernier n'arrive plus à contenir le mal-être du roi qui s'est désormais tourné en jalousie ouverte contre son ancien favori ; 1 S 19,9-24 répète l'épisode de l'attentat (versets 9-10) à la suite duquel David prend la fuite de la cour du roi avec l'aide de Mikal. À la fin de ce chapitre, lorsque Saül poursuit David à Rama, il est une dernière fois saisi par un esprit divin : alors que le texte n'explicite pas s'il s'agit du même esprit mauvais que dans les épisodes précédents, il est clair que ses effets sont néfastes car Saül s'abandonne à un état de transe incontrôlée et honteuse qui se termine par l'écroulement du roi nu sur le sol. À partir de ce moment-là, le déclin de Saül devient inéluctable. Comme cela a été observé depuis longtemps, cette série de récits est centrée sur le motif des esprits et se trouve encadrée par ce même motif, sur lequel se construit également le lien rédactionnel avec la section précédente en $1 \mathrm{~S} 16,13^{61}$. La série narrative qui se conclut au chapitre 19 doit, enfin, être complétée par l'épisode de la pre-

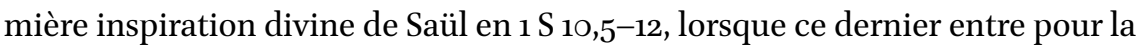
première fois dans un état de transe prophétique ${ }^{62}$. Ces récits apportent une contribution fondamentale à la compréhension du fonctionnement des esprits pour plusieurs raisons. D'abord, ils montrent que la possession est une composante essentielle de la prophétie en Israël ancien, même si elle est difficile à gérer et n'est jamais exempte de risques. En outre, ils mettent en contraste

6o Voir respectivement Num 11,25-29; 1 R 18,12 ; 2 R 2,9-10, $15^{-16 .}$

611 S 16,13 a-b : «Et Samuel prit la corne d'huile et lui [scil. David] donna l'onction au milieu

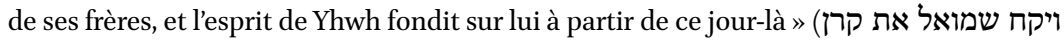
(השמן וימשח אתו בקרב אחיו ותצלח רוח יהוה אל דוד מהיום ההוא (הoir, par exemple, Stolz 1981, p. 109. Sur les aspects rédactionnels, voir également Dietrich 2015, p. 192-200 et 253-255; Blischke 2019, p. 18-24.

62 Sur la relation de ce passage avec le reste du corpus, voir Nihan 2006. Puisque toutefois la

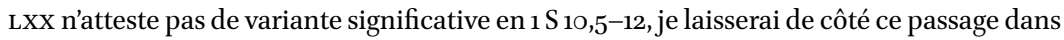
la comparaison entre hébreu et grec. 
l'action de l'esprit de Yhwh avec l'influence de l'esprit mauvais, tout en indiquant que seulement un esprit à la fois peut posséder une personne (lorsque l'esprit de Yhwh part de Saül, l'esprit mauvais arrive et vice-versa). De plus, dans la mesure où et l'inspiration divine provenant de Yhwh et l'esprit mauvais fonctionnent par la possession, ce texte suggère qu'il n'existe pas de différence substantielle entre l'esprit de Yhwh et les autres esprits, qui se distinguent seulement par leurs résultats. Enfin, l'épisode de l'esprit mauvais encadre le récit de l'ascension de David, qui est présenté avec des qualités royales dès sa première apparition en $1 \mathrm{~S} 16,18$, où il est décrit comme étant vaillant guerrier, intélligent, beau et avec Yhwh à ses côtés. Dans ce contexte, le contrôle des esprits apparaît comme une vertu royale et le contraste entre Saül et David se joue également sur cet aspect : alors que Saül est à la merci des esprits, David est capable de les maîtriser. À cet égard, $1 S_{16-19}$ atteste d'une tradition d'exorcisme par la musique, ici attribuée à David, qui aura une certaine fortune dans la tradition juive ancienne.

Lorsque nous lisons la version de l'histoire rapportée par le TM, nous remarquons deux points problématiques. Premièrement (1), les effets produits par l'esprit mauvais sur Saül ne sont pas décrits de manière explicite ni cohérente. En 1 S 16,14-16 l'hébreu utilise le verbe $b^{\prime} t$ («tourmenter», «terroriser»); en $1 \mathrm{~S}$ 18,10-11 l'esprit induit en Saül un état de transe (wayyitnabbé') qu'il n'arrive pas à gérer et qui semble engendrer une impulsion à tuer David par la lance ; en 1 S 19,9-10 il n'y a plus de référence à la transe, seule l'impulsion meurtrière du roi envers David est mentionnée. Ces inconsistances trahissent en partie la nature rédactionnelle de certains passages, que je discuterai dans la suite.

Deuxièmement (2), et comme je l'ai dit plus haut, la distinction entre l'esprit de Yhwh et l'esprit mauvais n'est pas toujours très claire. Nous pouvons comparer, à cet égard, le verset 14 avec le verset 23 (voir le tableau 8 ci-dessous). Dans le verset $14 \mathrm{~b}$, la préposition $m$ 't indique clairement que l'esprit mauvais est envoyé par Yhwh (mèètyhwh): par conséquent celui-ci semble être distinct de rûah yhwh nommé en 14a. Par contre, la même distinction est beaucoup moins évidente dans les versets qui suivent : les versets $15^{-16}$ comportent l'expression rûah 'êlōhim rā'âa, dans laquelle on pourrait comprendre 'êlōhîm comme signifiant «d'origine divine », alors qu'au verset 23a on parle seulement de rûah 'élōhîm. Enfin, à la fin du chapitre 19, l'esprit divin (rûah 'êlōhîm) s'empare une dernière fois de Saül avec des effets néfastes ${ }^{63}$.

Or le texte de la LXX présente des variantes significatives par rapport à ces deux points. Avant de les analyser, un court préambule sur la situation manuscrite s'avère encore nécessaire. Dans les livres de Samuel, l'analyse du grec est compliquée par une situation textuelle difficile, où l'histoire textuelle est

$63 \quad 1 S_{19,23-24 .}$ 
inséparable de l'histoire littéraire et même rédactionnelle, de manière encore plus complexe que dans le livre des Rois. Alors que la majorité des chercheurs reconnaît l'origine tardive du $\mathrm{TM}$, en grec plusieurs traditions sont attestées. À l'inverse de $1 \mathrm{R} 22$, le texte $\mathrm{B}$ est considéré, pour cette section, comme le meilleur représentant de la LXX ancienne ${ }^{64}$; la tradition hexaplaire est conservée par l'Alexandrinus (A) ainsi que par d'autres minuscules ${ }^{65}$; nous avons, enfin, le texte lucianique $(\mathrm{L})^{66}$, qui réunit à son tour au moins deux traditions textuelles, à savoir des révisions plus tardives et un texte "proto-lucianique » qui, comme nous l'avons vu plus haut, peut contenir, parfois, des variantes anciennes. De manière générale et en comparaison avec 1 Rois, l'apport du texte lucianique pour cette section des livres historiques est considéré comme moins important en vue de la reconstruction de la LXX ancienne; la comparaison entre $\mathrm{B}$ et $\mathrm{L}$ reste néanmoins utile pour établir des développements internes aux traditions grecques.

Une des conséquences de cette complexité pour mon analyse est le fait que 1S 18,10-11 est absent en B. L'hébreu offre plusieurs indices de la nature rédactionnelle et secondaire du passage : en 1 S 18,11, on dit que David évite par deux fois la lance de Saül, même si le deuxième épisode ne sera raconté qu'au chapitre suivant. De plus, la référence à la transe de Saül en 1 S 18,10 (wayyitnabbe’') comme effet de l'esprit mauvais semble anticiper la transe incontrôlée dont il fait l'objet en $1 S$ 19,19-24. Il me paraît, ainsi, clair que l'absence de ces versets dans la LXX ancienne remonte forcément à la Vorlage du traducteur ${ }^{67}$. Le texte lucianique, quant à lui, atteste des variantes secondaires qui concernent l'action de l'esprit en $14 \mathrm{~b}$ et la relation de l'esprit avec la divinité. Elles ont, néanmoins, un certain intérêt, comme le montre le tableau suivant, qui récapitule les mentions des esprits en $1 \mathrm{~S} 16$ :

64 Pour cette section, le texte B est représenté par le Vaticanus et par le manuscrit y (= 121), ainsi que par les versions éthiopiennes. Pour une introduction récente, claire et exhaustive au texte de 1 Samuel, voir Kauhanen 2012. Voir également Bernard Grillet et al. (1997, p. 9-120), qui, toutefois, est antérieur à la publication complète des fragments retrouvés à Qumrân par Cross, Parry, Saley et Ulrich (Cross et al. 2005, DJD 17).

65 Mss. 247, 376.

66 Pour les manuscrits du texte L, voir supra, p. 239, note a.

67 La majorité des chercheurs estime qu'en i S 18 la LXX ancienne lit un texte court dans l'hébreu; Stephen Pisano (1984) a, en revanche, essayé de démontrer l'antériorité du TM par rapport à la LXX : $1 \mathrm{~S}$ 18,10-11, tout comme les autres passages de ce chapitre qui ne sont pas attestés en B, aurait été éliminé intentionnellement dans le grec notamment à cause de sa redondance. Cette démarche serait à comprendre comme faisant partie d'un processus d'assouplissement qui commence déjà en hébreu (voir ibid., p. 86). Le volume de Barthélemy, Gooding, Tov et Lust (Barthélemy et al. 1986) sur 1 S 17-18 a apporté une série d'éléments qui font plutôt pencher pour le contraire. Une question séparée, qui ne peut pas être discutée ici, est, en revanche, celle du rapport entre la version longue et la version courte du texte hébreu. 
TABLEAU 8 Esprits en 1 Samuel 16

\begin{tabular}{llll}
\hline $\mathrm{S} 16$ Hébreu & $\operatorname{Lxx}(\mathrm{B}) \quad \mathrm{L} \quad$ Autres & $\begin{array}{l}\text { Aquila/ } \\
\text { Symm/Th }\end{array}$
\end{tabular}

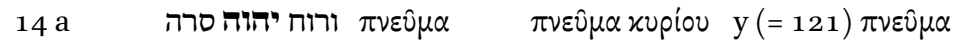
xupiou $\dot{\alpha} \pi \dot{\varepsilon} \sigma \tau \eta \quad \theta \varepsilon \circ \hat{0}$
Et l'esprit de $\quad \alpha \pi \varepsilon \dot{\varepsilon} \sigma \eta$
Yhwh s'en alla
de Saül

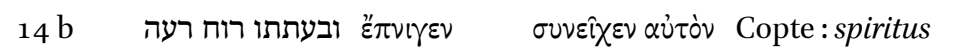

\begin{tabular}{|c|c|c|c|}
\hline מאת יהוה & $\alpha u ̉ \tau \dot{v}$ & $\pi \nu \varepsilon \hat{\mu} \mu \alpha$ & malus \\
\hline Un esprit & $\pi \nu \varepsilon \hat{\mu} \mu \alpha$ & 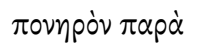 & Origène (ms \\
\hline mauvais venu & Tounpòv & xupiov, xai & grecs) : $\pi \nu \varepsilon \hat{\mu} \mu \alpha$ \\
\hline de Yhwh le & $\pi \alpha p \dot{\alpha}$ xupiov & 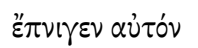 & 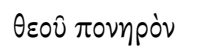 \\
\hline
\end{tabular}

$A: \dot{\varepsilon} \theta \alpha \mu \beta \varepsilon l$

tourmenta

15

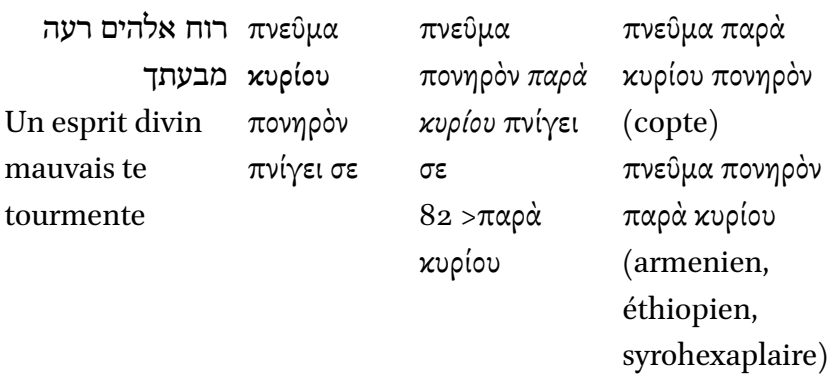

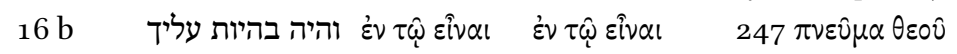

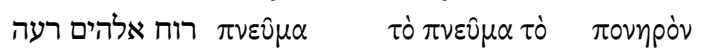

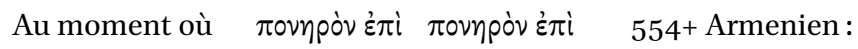

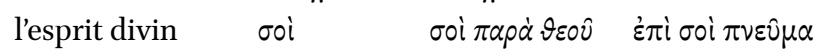

mauvais sera $\quad \pi$ ovnpòv $\pi \alpha \rho \dot{\alpha} \theta \varepsilon \circ \hat{0}$

sur toi Autres :

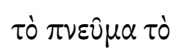

тovnpòv ou

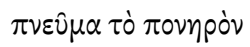


TABLEAU 8 Esprits en 1 Samuel 16 (cont.)

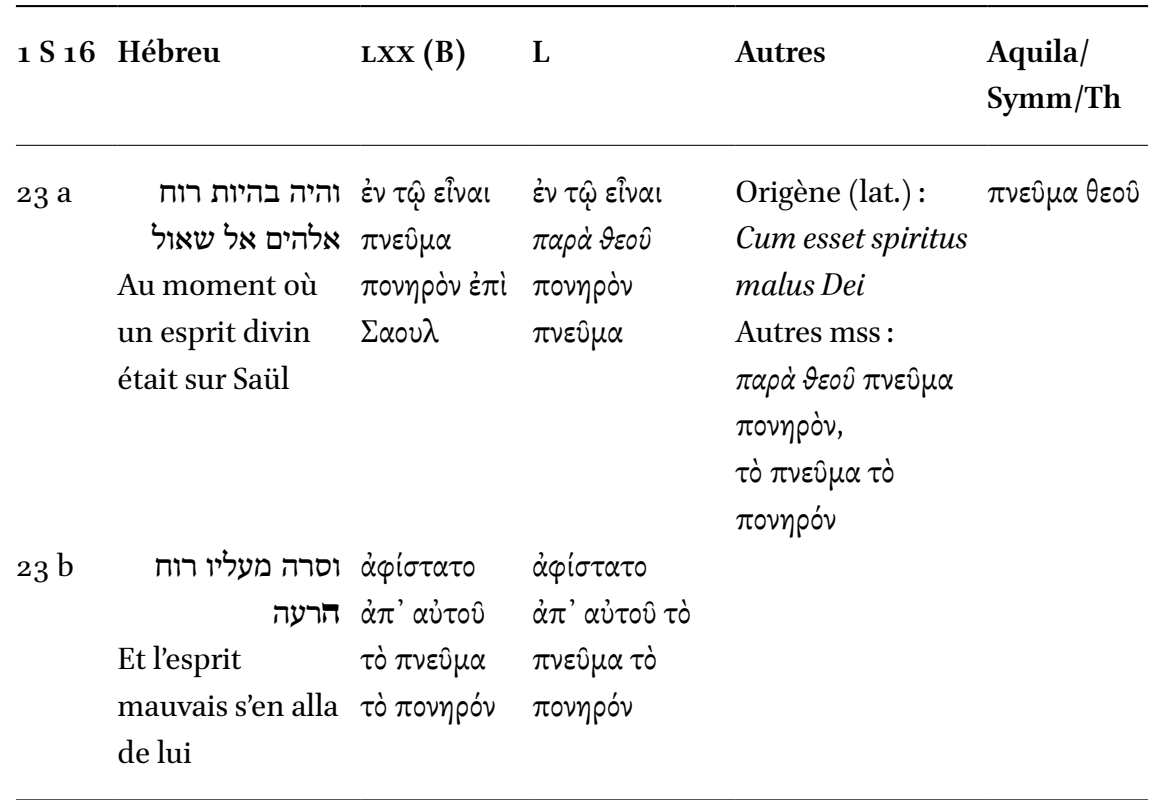

Le tableau 8 montre plusieurs différences dans les traditions grecques par rapport au TM, dont deux sont particulièrement significatives. Premièrement (1), au verset 14b le verbe $b^{\prime} t$, «saisir par la peur», «terroriser», «tourmenter», est rendu par $\pi v i \gamma \omega$, « étouffer», terme typique de la littérature médicale, avec une équivalence qui n'est jamais attestée ailleurs dans la LXX pour $b^{\prime} t$. En conformité à ce choix, au verset 23 l'expression ורוח לשאול (warāwah lašāâul,

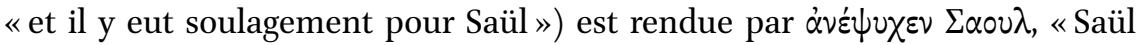
reprit haleine » : cette traduction rend explicite le lien entre l'usage du verbe $r w h$ et le souffle qui, très vraisemblablement, était déjà présent en hébreu68. Nous avons là un premier témoignage de l'interprétation de l'attaque de l'esprit en termes physiologiques et médicaux, à savoir comme un étouffement. Une telle interprétation n'est pas surprenante si l'on tient compte du fait que l'étranglement est une des actions attribuées par excellence aux démons déjà en Mésopotamie. En comparant B avec L au verset 14b, nous observons dans la tradition lucianique une tendance à expliciter les différentes phases de l'action

68 Voir Dietrich (2015, p. 23) qui traduit: « und dann konnte Saul aufatmen ». Pour la relation du verbe $\alpha \nu \alpha \psi v ́ \chi \omega$ avec la respiration, voir Jouanna 1987, p. 210-211 et Grillet et al. 1997, p. $288-289$. 
de l'esprit, qui d'abord saisit Saül ( $\sigma \nu v \varepsilon i ̂ \chi \varepsilon v)$ et ensuite l'étouffe ( $\varepsilon$ $\pi v \vee \gamma \varepsilon v)$. Vu que Symmaque opte également pour $\sigma$ $v \varepsilon i \chi £ \varepsilon v$, il est probable que le texte grec ait ici été corrigé sur la base des révisions hébraïsantes. Toutefois, Tuukka Kauhanen n'exclut pas l'hypothèse selon laquelle cette interprétation serait antérieure à la révision lucianique car elle est déjà attestée chez Tertullien dont l'œuvre précède le travail des réviseurs ${ }^{69}$. Deuxièmement (2), le texte grec semble trahir un effort pour distinguer plus nettement l'esprit de Yhwh de l'esprit mauvais car ce dernier n'est plus défini comme divin : aux versets $16 \mathrm{~b}$ et 23 a, B a seulement $\pi v \varepsilon \hat{u} \mu \alpha \pi$ ovnpóv ${ }^{70}$. Cette tendance, déjà attestée en $B$ et relevant donc, vraisemblablement, de la LXx ancienne, est ensuite prolongée dans les traditions grecques plus tardives préservées par L, qui traduisent elohîm par $\pi \alpha \rho \dot{\alpha} \theta \varepsilon \circ \hat{v}$, en conformité avec verset $14 \mathrm{~b}$. Le texte $\mathrm{L}$ tient à souligner que l'esprit mauvais agit dépendamment de Yhwh, en rapprochant ainsi la traduction grecque du texte massorétique et en supprimant, en même temps, toute possibilité d'identification entre l'esprit de Yhwh et l'esprit mauvais.

Une différence ultérieure (3) est détectable en $1 S_{16}$,18, lorsqu'un des domestiques présente David au roi comme un jeune homme doué pour la musique: « effectivement j'ai vu un fils de Jessé le Bethléémite, il sait jouer ». Ici l'expression yōdēa' naggèn «il connaît l'art de jouer (un instrument à cordes)» est

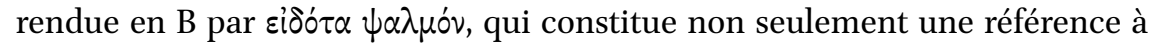
la tradition d'exorcismes opérés par la musique, mais à David comme auteur du psautier ${ }^{71}$. Cette tradition est encore appuyée par Aquila au verset 16 et par Théodotion, au verset 23, qui rendent respectivement bakkinnôr et 'et hakkinnôr (kinnôr étant le nom de l'instrument à cordes joué par David) par $\psi \alpha \lambda \tau \tau^{p} ı$ ov. Enfin, nous pouvons repérer un dernier élément (4) de nouveauté dans le récit de l'attaque de Saül contre David, en 1 S 19,9:

ותהי רוח יהוה רעה אל שאול והוא בביתו יושב וחניתו בידו ודוד מנגן ביד

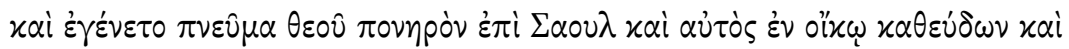

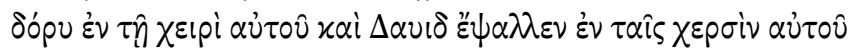

69 Kauhanen 2012, p. 92-95.

70 Le choix de l'adjectif $\pi$ ounpós au lieu de $x$ axós ne semble pas véhiculer une signification particulière. D'après Richard Trench (1855, p. 41-43) dans le Nouveau Testament rovnpós exprime parfois une perversité plus grave que $x \alpha$ ó́s. Dans la LXx, les deux adjectifs sont employés pour traduire la quasi-totalité des adjectifs $r^{\prime}, r^{\prime \prime}$ et $r^{c} h$, de manière assez inter-

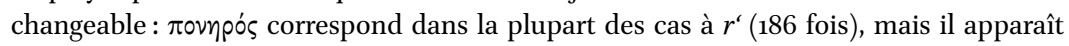
néanmoins 63 fois comme équivalent de $r^{c} h$. Vice-versa, xaxós traduit 70 fois $r^{`}$ et 138 fois r'h (Dodd 1954, p. 75).

Pour un commentaire à ce sujet, voir Grillet et al. 1997, p. 29 o. 
Et un esprit mauvais de Dieu fut sur Saül. Il dormait, dans sa maison, et il avait une lance à la main, et David pinçait de ses mains les cordes.

À la différence du TM, l'esprit saisit Saül dans son sommeil : $\varkappa \propto \theta \varepsilon v ́ \delta \omega$, « dormir », correspond ici exceptionnellement à yôšēb en lisant probablement le verbe

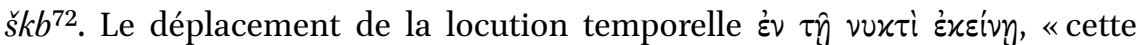
nuit-là », de la fin du verset 10 au début du verset 11, confirme également que, d'après le traducteur grec, la tentative d'homicide a lieu pendant la nuit et que, suite à cet événement, David prend immédiatement la fuite. Ce changement de scénario du jour à la nuit confère, certes, un aspect plus dramatique à l'action ${ }^{73}$, mais la situe également dans un moment très favorable aux attaques démoniaques.

Essayons à présent de tirer quelques conclusions des comparaisons entre texte hébreu et texte grec. Lorsque l'on considère l'apport des versions grecques pour comprendre $1 \mathrm{R} 22$, nous voyons que les esprits, tout en restant des puissances subordonnées à Yhwh, ont, dans la Bible, une agentivité plus prononcée que ce que l'on pense d'habitude. Néanmoins cette agentivité n'est efficace que si elle passe, en quelque sorte, par l'appui du dieu: de ce point de vue, il importe de relever qu'il n'y a pas d'opposition entre version hébraïque et version grecque, même si dans la tradition grecque attestée par le texte lucianique, qui pourrait être assez ancienne, cette interaction est plus développée. La capacité d'initiative des esprits est considérable, mais leur autonomie reste assez relative, ce qui est en lien avec le fait que les esprits ne sont pas à compter parmi les puissances majeures de l'assemblée céleste. En 1 S 16-19, alors que le texte hébreu consent à une certaine ambiguïté concernant l'identité de l'esprit mauvais et celle de l'esprit de Yhwh, les versions grecques tendent à progressivement les distinguer; elles donnent, en outre, une interprétation médicale de l'action de l'esprit comme s'assimilant à un type d'étouffement et, en 1 S 19, suggèrent que la dernière attaque de l'esprit saisit le roi dans son sommeil, pendant la nuit. Une telle caractérisation de l'esprit mauvais rapproche considérablement la possession par l'esprit d'une attaque démoniaque.

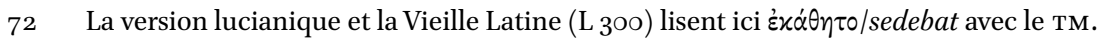

73 Dans le fragment préservé à Qumrân $\left(1 Q 5^{2}=4 Q^{2}{ }^{2}{ }^{b}\right.$, fr. 5, 2), la locution temporelle est à la même place que dans la Lxx et il est fort possible que, dans ce cas, la LXX et les manuscrits qumrâniens reflètent la position originelle (ainsi Cross et al. 2005, DJD 17, p. 229). Malheureusement, le verset précédent n'a pas été préservé: on ne sait donc pas si l'épisode de l'attaque de Saül sur David dans la version de Qumrân eut lieu pendant la même nuit. Voir Grillet et al. 1997, p. 319. 
Avant d'examiner les autres passages qui complètent l'analyse des esprits dans la LXx, il apparaît nécessaire de revenir sur l'équivalence entre l'hébreu rûah et le grec $\pi v \varepsilon \hat{u} \mu \alpha$ qui traduit rûah dans la plupart des occurrences. Cette correspondance a reçu une certaine attention dans les études sur la pneumatologie du premier christianisme, notamment car $\pi v \varepsilon v \hat{\mu} \alpha$ devient un terme clé de la sotériologie chrétienne ${ }^{74}$. Toutefois, comme dans le cas de rûah dans la Bible hébraïque, l'attention a plus été portée sur «l'esprit» de Yhwh que sur «les esprits », privilégiant une approche théologique plutôt qu'historico-religieuse. Le dossier mérite donc d'être rediscuté selon ce que j'appellerais une perspective "plurielle», qui peut, à mon avis, apporter de nouveaux éléments à la discussion. Les questions ayant dominé le débat jusqu'à présent portent sur les influences réciproques entre les usages hébreu et grec du terme. Une première hypothèse $(1 \mathrm{a})$ envisage une influence du rûaḥ hébreu sur le concept du $\pi v \varepsilon \hat{\mu} \mu \alpha$ grec: le sens psychique dans lequel nous avons vu que rûah pouvait être employé aurait été imposé au $\pi v \varepsilon v ̂ \mu \alpha$ grec, qui omet ce sens, afin que ce dernier corresponde à son équivalent sémitique ${ }^{75}$. Un deuxième volet de la même hypothèse $(1 \mathrm{~b})$ considère que la $\mathrm{Lxx}$ a joué un rôle fondamental dans l'introduction d'un nouveau concept: «esprit de dieu ( $\pi v \varepsilon v \hat{\mu \alpha} \theta \varepsilon \circ \hat{)})$, notion qui aurait été étrangère à la culture grecque, laquelle préfère d'habitude d'autres termes pour décrire des puissances divines incorporelles ou indéterminées, tels que $\delta \alpha i \mu \omega \nu$ ou $\tau \dot{x} \chi \eta^{76}$. Vice-versa, une deuxième hypothèse (2) insiste sur le rôle du grec dans l'introduction de nouvelles significations pour le rûah biblique. Selon David Hill, l'idée des esprits comme entités indépendantes de Yhwh se serait développée à partir de la conceptualisation grecque du $\pi v \varepsilon \hat{\mu} \mu \alpha$ comme substance, idée qui serait, à son tour, étrangère à l'hébreu ${ }^{77}$. En réalité, cette hypothèse vient d'être contredite dans la section précédente, où nous avons vu que les esprits, tout en étant pour la grande majorité d'origine divine, ne sont pas de pures et simples émanations de la divinité, mais des entités différenciées de Yhwh déjà dans le texte hébreu ${ }^{78}$. Quant à la première hypothèse, elle demande de reprendre l'analyse du champ sémantique de $\pi v \varepsilon \hat{\mu} \mu \alpha$ ainsi que les rapports avec ses possibles concurrents, à savoir d'autres

74 Parmi les études dans le domaine du Nouveau Testament qui prennent sérieusement en compte la LXX, on peut voir Schoemaker 1904; Hill 1967 ; Isaacs 1976 ; M. Edwards 2012.

75 Schoemaker 1904; Hill 1967.

76 Isaacs 1976, p. 16-17, suivie par M. Edwards 2012.

77 Hill 1967, p. 202-220.

78 Voir, sur ce point, la critique d'Isaacs 1976, p. 14. 
équivalents grecs qui auraient pu être choisis, mais qui, pour une raison ou une autre, ont été écartés par les traducteurs lors de la « compétition sémantique ».

Il faut commencer par rappeler que $\pi \nu \varepsilon \hat{\nu} \mu \alpha$ entretient avec la racine $\pi \nu \varepsilon \hat{\imath}$, « souffler », « respirer », « haleter », mais aussi « dégager une odeur », un rapport similaire à celui de rûah avec le verbe $r w h$. Пveîv décrit chez Homère le souffle du vent, mais également la respiration et, donc, la vie ${ }^{79}$. Le substantif, quant à lui, apparaît pour la première fois probablement chez Archiloque ${ }^{80}$ et se retrouve plus fréquemment en prose qu'en poésie. Un deuxième dérivé

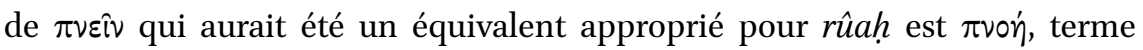
homérique qui indique autant le souffle que l'air ${ }^{81}$. Au vu de leur signification primaire d'« air en mouvement », $\pi \nu \varepsilon \hat{u} \mu \alpha$ et $\pi \nu \circ \eta$ peuvent indiquer à la fois l'haleine et donc la vie, en se superposant partiellement à $\psi v \chi \eta^{\prime}$, et le vent, en étant alors synonymes de $\alpha v \varepsilon \mu \circ \varsigma$. Concomitamment, $\pi v \varepsilon \hat{\mu} \mu \alpha$ et $\pi v o^{\prime}$ apparaissent

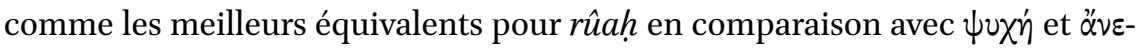

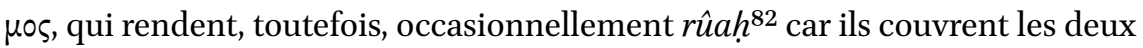
domaines sémantiques.

Conformément à son usage en grec classique, $\pi v 0$ ý est utilisé dans la LXX surtout comme équivalent de nəšâmâ, terme assez rare lui aussi et surtout poétique, partiellement synonyme de rûah et parfois utilisé en parallèle à celui-ci ${ }^{83}$. Cette équivalence est établie déjà par le traducteur de la Genèse, en Gen 2,7

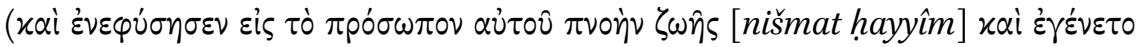

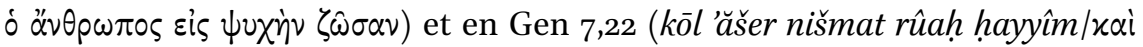
$\pi \dot{\alpha} \nu \tau \alpha$ ö $\sigma \alpha$ है $\chi \varepsilon ı ~ \pi \nu \circ \eta े \nu ~ \zeta \omega \eta ิ \varsigma)$. En revanche, il n'est pas inutile d'observer que dans les inscriptions funéraires d'époque hellénistique une des expressions pour

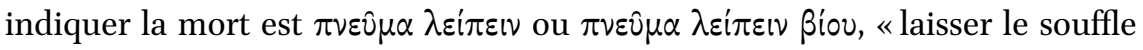
de la vie » ou simplement «laisser le souffle», qui peut être comprise comme « expirer le dernier souffle» mais également comme «laisser la vie, le principe vital ${ }^{84}$. Cet usage est également attesté sur quelques épitaphes juives de l'Égypte gréco-romaine ${ }^{85}$. Lídée du $\pi v \varepsilon \vartheta \hat{\mu} \alpha$ comme force vitale ainsi que

79 Par exemple Hom., Il. 17, 447 ; Od. 4, 361 et $457 ; 18$, 131, voir J.H. Schmidt 1876 , no. 55 .

$80 \quad$ Arch., $\mathrm{S} 478 \mathrm{~b}$ Page (Supplementum) : la lecture est restaurée par les éditeurs du papyrus.

81 Пvớ traduit rûah en Ez 13,13; Is 38,16; Prov 1,23; 11,13.

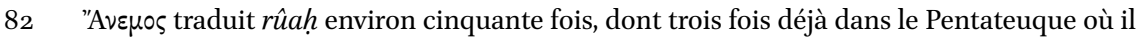

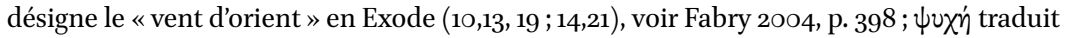

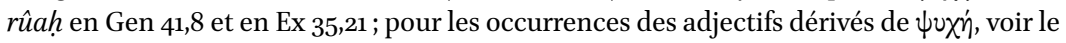
tableau 6.

83 Job 27,$3 ; 32,8 ; 33,4$; Is 42,$5 ; 57,16$.

84 Par exemple (liste non exhaustive), Attique: IG II $^{2} 5227 \mathrm{a}, I G \mathrm{II}^{2} 5255$; Grèce centrale: IG IX 1,873, IG IX 1,164; Cnide : SE G 6o.1122 ; Asie Mineure : $S E G$ 6.414; Égypte : SE G 24.1239; Italie : IGUR III 1381 .

85 Bernand 1969, no. 37, p. 184-189, surtout p. 188 pour la restitution du mot $\pi v \varepsilon \hat{\mu \alpha}$. 
la proximité entre $\pi \nu \varepsilon \hat{\mu} \mu \alpha$ et $\psi v \chi \eta \dot{~ n e ~ s o n t ~ p a s ~ d e s ~ i n n o v a t i o n s ~ h e l l e ́ n i s t i q u e s ~}$ car elles sont déjà attestées chez les philosophes présocratiques, notamment chez les philosophes «naturalistes » de l'école milésienne ${ }^{86}$ et probablement chez les atomistes également ${ }^{87}$. La présence de $\pi v \varepsilon v \hat{\mu} \alpha$ parmi les inscriptions suggère, néanmoins, qu'à l'époque hellénistique le lien sémantique entre $\pi \nu \varepsilon \hat{u} \mu \alpha$ et la vie individuelle a franchi les frontières du langage purement philosophique et littéraire pour faire, désormais, partie du langage religieux courant.

Lautre grand domaine où l'idée du $\pi v \varepsilon v \hat{\mu} \alpha$ comme substance essentielle à la vie se développe est celui de la littérature médicale. Le principe du $\pi \nu \varepsilon \hat{\mu} \mu \alpha$ comme un «air inné » est déjà énoncé dans les traités hippocratiques. L'idée d'une substance à la fois matérielle et invisible qui peut présider aux fonctions psychiques est ensuite développée par la physiologie aristotélicienne ${ }^{88}$ ainsi que par la médecine hellénistique, notamment par l'école d'Alexandrie qui comptait parmi ses membres les illustres Hérophile de Chalcédoine et Érasistrate de Céos dont la doctrine nous intéresse tout particulièrement ici89. Érasistrate estime que le $\pi \nu \varepsilon \hat{\nu} \mu \alpha$ est une substance aérienne différente de l'air extérieur, qui remplit les poumons et dont la moitié passe dans les artères

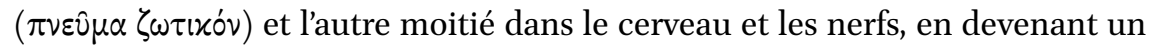

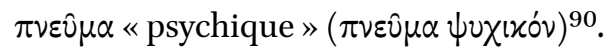

C'est précisément de cette double tradition philosophique et médicale que va dériver l'idée des stoïciens du $\pi \nu \varepsilon \hat{\mu} \alpha$ comme véritable puissance divine qui

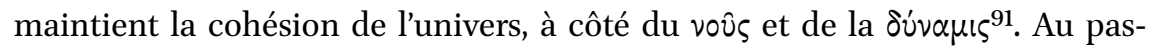
sage, ces deux termes sont attestés dans la LXX : vôิ surtout comme équivalent de לבב/לב (littéralement «cœur», mais aussi «intériorité», «volonté»,

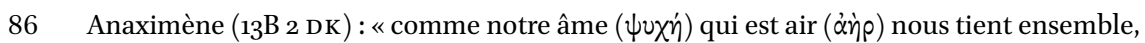
ainsi le souffle $(\pi v \varepsilon \hat{v} \mu \alpha)$ et l'air ( $\dot{\varkappa} \hat{\jmath})$ tiennent ensemble le monde entier ».

87 D'après le témoignage de Macrobe, Démocrite décrivait lâme comme un esprit rempli d'atomes (68A 103 DK). Voir, sur ce point, Pralon 2015, p. 6. Diogène Laërce attribue également à Xénophane l'idée que l'âme soit $\pi v \varepsilon \hat{\mu} \mu \alpha$ (D.L. 9, 19, 10-11).

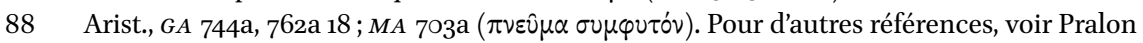
2015 , p. $7-8$.

89 L'œuvre de ces auteurs ne nous est parvenue que par tradition indirecte, transmise surtout par Celse et Galien, et en partie par les philosophes stoïciens. Des fragments d'un «Traité sur la fièvre » préservé par un papyrus d'Héracléopolis ont été provisoirement attribués à Érasistrate ou à un élève de son école. Le texte contient effectivement une référence au $\pi \nu \varepsilon \hat{\mu} \mu \alpha$ qui passe dans les artères (P. Köln. 8 327, col. iii 20-25, édité par Gronewald, Maresch et Römer, 1997).

$90 \quad$ Gal., De placit. Hipp. et Plat. (p. 245 Müller). Voir Verbeke 1945, p. 175-191 ; L. Wilson 1959; sur les aspects «matériels » de cet air dans le débat philosophique, voir Lloyd 2007.

91 La doctrine pneumatique est explicitement attribuée à Zénon, le fondateur de l'école: voir, par exemple, D.L. 7, 156-157. Pour Chrysippe, voir SVF 2, 473 (= Alex. Aphr., Mixt. 7, $216,14-17)$. 


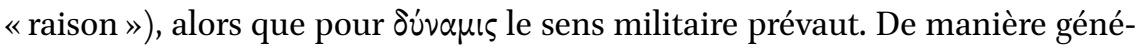
rale, la convergence entre savoir philosophique et savoir médical ne doit pas surprendre car, généralement, dans l'Antiquité, et en Grèce ancienne plus particulièrement, philosophie et médecine ne sont que deux volets d'une même enquête sur la nature. En partant d'une conception du monde fondée sur un modèle physiologique qui reproduit un organisme vivant, les stoïciens développent ainsi, de leur côté, l'idée d'une identité commune entre $\pi \nu \varepsilon \hat{\mu} \mu \alpha$ et $\psi v \times \dot{\eta}^{92}$.

En revanche, l'idée que $\pi v \varepsilon \hat{\mu} \alpha$ ne puisse pas décrire l'inspiration divine en Grèce n'est pas corroborée par un examen attentif des sources. L'usage de ce mot pour décrire l'inspiration poétique est connu dès l'époque classique, à

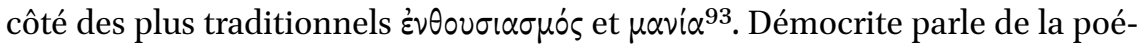
sie comme d'un travail inspiré par l'enthousiasme, au sens propre du terme, ou par «un souffle sacré ${ }^{94}$. Le «souffle» peut décrire également l'activité prophétique. Dans Prométhée enchaîné d'Eschyle, la jeune Io décrit son malheur dans un langage qui reprend celui de la possession oraculaire et affirme se sentir emportée par un «souffle de folie fou »; dans l'Agamemnon, Cassandre parle d'Apollon qui « souffle » sur elle avec force ${ }^{95}$. À partir de l'époque hellé-

92 Pour Zénon, voir, par exemple, $s V F$ 1, 136 (= Gal., [Hist. Phil.] 24); pour Chrysippe, $s V F$ 2, 885 (Gal., De placit. Hipp. et Plat. 2,1, p 170, 9-10); sur $\pi v \varepsilon \hat{\mu \alpha} \alpha$ comme puissance divine chez Chrysippe, voir D.L. 7, 139; Aët. 1, 7, $33=S V F 2$, 1027). Sur la psychologie pneumatique du premier stoïcisme ainsi que sur ses évolutions, le travail de Gérard Verbeke (1945), bien que daté, est encore la meilleure référence. Voir également les sources discutées par Pralon 2015, p. 11-18. Certaines de ces sources sont également rassemblées par Levison 2009, p. 137-140 et citées rapidement par Edward 2012, p. 83-84.

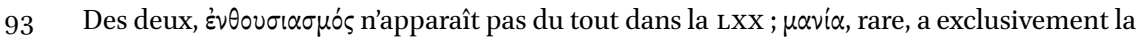
connotation péjorative de «folie », qui est également saillante en grec (Os 9,7, 8 ; Ps 40,5; Sap 5,$4 ; 4$ Mac 8,$5 ; 10,13)$.

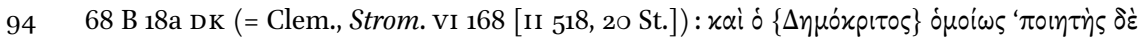

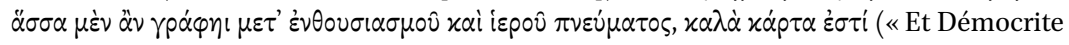
dit de la même manière : c'est vraiment beau toute œuvre qu'un poète écrit par inspiration ou souffle divin »). Sur la nature divinement inspirée de la parole poétique comparer également avec $68 \mathrm{~B} 17$ et $21 \mathrm{DK}$.

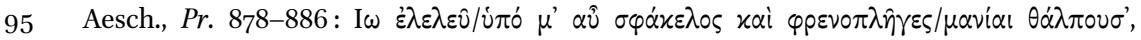

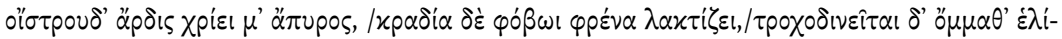

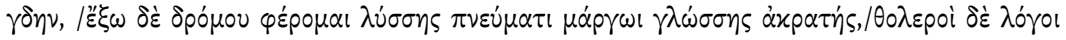

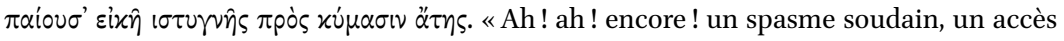
délirant me brûlent. Le dard du taon me taraude, tel un fer rougi. Mon cœur épouvanté piétine mes entrailles. Mes yeux roulent convulsivement. Emportée hors de la carrière par un furieux souffle de rage, je ne commande déjà plus à ma langue, et mes pensées confuses se heurtent en désordre au flot montant d'un mal hideux» (tr. P. Mazon, Paris,

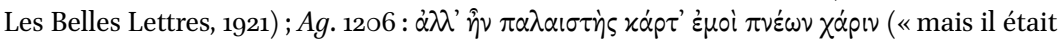
un lutteur qui soufflait [sa] grâce contre moi »). 
nistique, $\pi v \varepsilon \hat{\mu} \mu \alpha$ semble être désormais entré dans le vocabulaire de la possession prophétique, notamment en lien avec l'oracle d'Apollon, et la nature précise de ce souffle, ou esprit, fait l'objet d'une discussion ${ }^{96}$. Le témoignage le plus détaillé est sans doute celui de Plutarque qui discute des phénomènes à la base de l'inspiration de la Pythie et parle d'un « flux prophétique et d'un

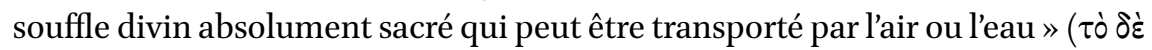

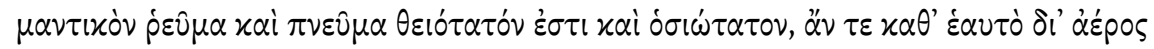

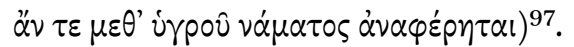

On peut, en outre, citer un fameux passage de Strabon, qui rapporte une rumeur selon laquelle la Pythie donne ses oracles en recevant un $\pi v \varepsilon v \hat{\mu} \alpha$

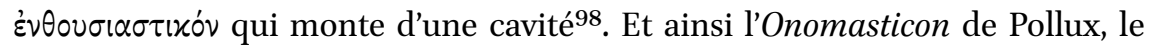
premier «thesaurus » de l'Antiquité, explique ce qu'est un esprit ou souffle prophétique : «si l'esprit est en quelque part prophétique, le lieu est plein du dieu, inspiré, possédé, sous l'influence divine et occupé par la divinité, tout comme l'homme qui donne l'oracle : celui-ci est inspiré, agité par le dieu, possédé et plein du dieu, et changé par le dieu. On pourrait appeler l'esprit également souffle divinatoire, haleine démonique, air divin, vent prophétique, voix d'invocation ${ }^{99}$. L'importance particulière qui devait être rattachée au « souffle » d'Apollon est encore confirmée par une inscription trouvée à Cyrène datant du début du $\mathrm{II}^{\mathrm{e}} \mathrm{s}$. de notre ère, sur la base d'un bas-relief qui devait représenter le dieu. La partie supérieure du relief est perdue : il ne reste que les pieds du dieu, le tripode et un griffon à côté de l'inscription. Le texte parle de la « vérité de l'esprit du dieu », vraisemblablement en se référant à son oracle ${ }^{100}$.

Comme l'ont justement souligné Joseph Fonterose et plus récemment Julia Kindt $^{101}$, en ce contexte, il ne faut pas comprendre la mention du $\pi v \varepsilon \hat{\mu} \mu \alpha$ comme une référence à des vapeurs ou à des exhalations méphitiques qui auraientintoxiquéla Pythie. Cetteinterprétation correspond àune construction

96 En complément aux passages discutés ici, on peut voir Arist. [Mu.] 395 b.

97 Plut., Def. orac. $43^{2 d}$.

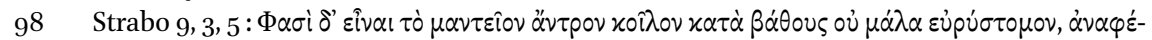

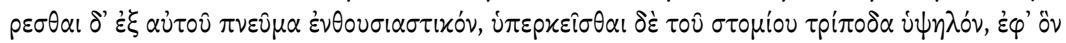

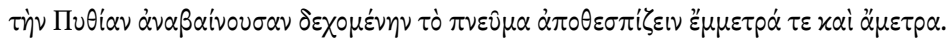

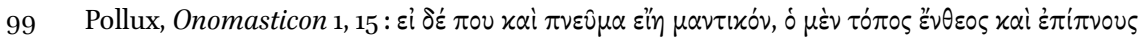

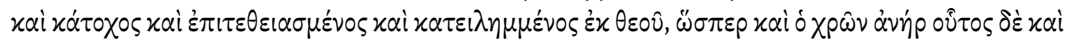

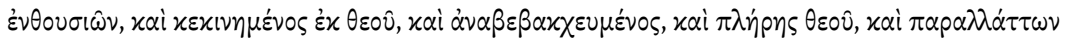

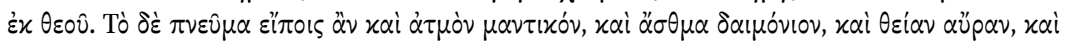

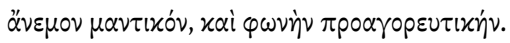

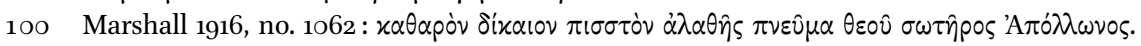
On peut comparer également le $\pi v \varepsilon \hat{\mu} \alpha \alpha$ $\theta \varepsilon \hat{o}$ v de l'oracle d'Apollon à Didyme, en Asie Mineure : IDidyma 159, l. 12 (fin III ${ }^{\mathrm{e}}$ siècle).

101 Fontenrose 1978, p. 197-202; Kindt 2016, p. 6. 
chrétienne, voire moderne, du fonctionnement de l'oracle. Par une articulation très similaire à celle mise en place par la physique et la cosmologie stoïciennes, le fait que le $\pi v \varepsilon \hat{\mu} \alpha \alpha$ soit conceptualisé dans ces sources comme une substance aérienne n'empêche pourtant pas le fait qu'il soit en même temps considéré comme une puissance émanant du dieu : en tant qu'indice d'une inspiration divine à l'œuvre, il est inséparable de la divinité elle-même. La position de l'aristotélicien Lamprias au cours du débat sur la disparition de l'oracle de la Pythie mis en scène par Plutarque vise, notamment, à préciser ce point ${ }^{102}$.

L'enquête sémantique sur $\pi \nu \varepsilon \hat{\mu} \mu \alpha$, dont les résultats sont visualisés en synthèse dans la carte ci- dessous (p. 258), laisse donc émerger ce mot comme étant le meilleur équivalent possible pour rûah car il recouvre les mêmes domaines sémantiques et active une série d'associations qui se recoupent avec celles de l'hébreu (notamment météorologiques et physiologiques). Même si d'autres termes couvrent des domaines sémantiques spécifiques (comme $\psi v x \eta \dot{~ e t ~ a ̀ v \varepsilon-~}$

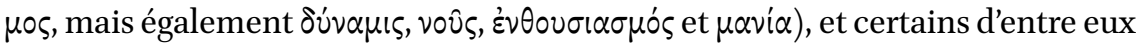
peuvent à l'occasion traduire rûah, aucun d'entre eux n'a une extension comparable à celle de $\pi v \varepsilon \hat{\mu} \mu \alpha$. En outre, rûah et $\pi \nu \varepsilon \hat{u} \mu \alpha$ partagent non seulement une métonymie sémantique puissante, à savoir celle entre souffle et vie, mais également la représentation du souffle comme une «substance » qui, tout en demeurant invisible, est douée d'une une certaine matérialité.

De plus, la documentation médicale et philosophique d'époque hellénistique montre que les aspects psychiques du $\pi v \varepsilon \hat{\mu} \mu \alpha$ ne sont pas absents de la pensée grecque. On peut, toutefois, se demander dans quelle mesure les premiers traducteurs avaient accès à ce type de littérature. À cet égard, il faut également rappeler que les traducteurs étaient conscients d'un déséquilibre entre les deux notions. Cela se remarque dans le tableau 6 dans les cas où les traducteurs, indépendamment les uns des autres, se détachent de l'équivalence habituelle entre rûah et $\pi v \varepsilon \hat{\mu} \alpha \alpha$ et lui préfèrent d'autres expressions qui décrivent des émotions ou des qualités humaines de manière moins ambiguë. À cet égard, une enquête rapide sur l'usage de $\pi v \varepsilon \hat{\mu} \mu \alpha$ dans le Pentateuque confirme que le mot relève de la même polysémie que rûah, exception faite des aspects psychiques, qui sont systématiquement traduits différemment: l'on peut citer

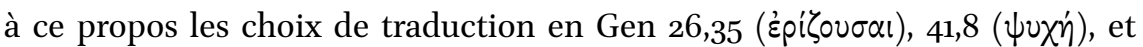

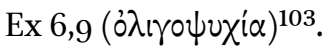

Une autre conséquence de l'analyse de ce dossier est que l'idée du $\pi \nu \varepsilon \hat{\nu} \mu \alpha$ $\theta \varepsilon o \hat{~ c o m m e ~ n o u v e a u t e ́ ~ i n t r o d u i t e ~ p a r ~ l a ~ L X X ~ d a n s ~ l a ~ p e n s e ́ e ~ g r e c q u e ~ a ̀ ~ p a r-~}$ tir d'un arrière-plan sémitique doit être nuancée, notamment au vu de la

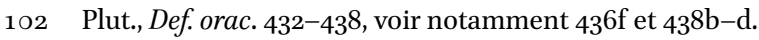

103 Voir supra, tableau 6. 


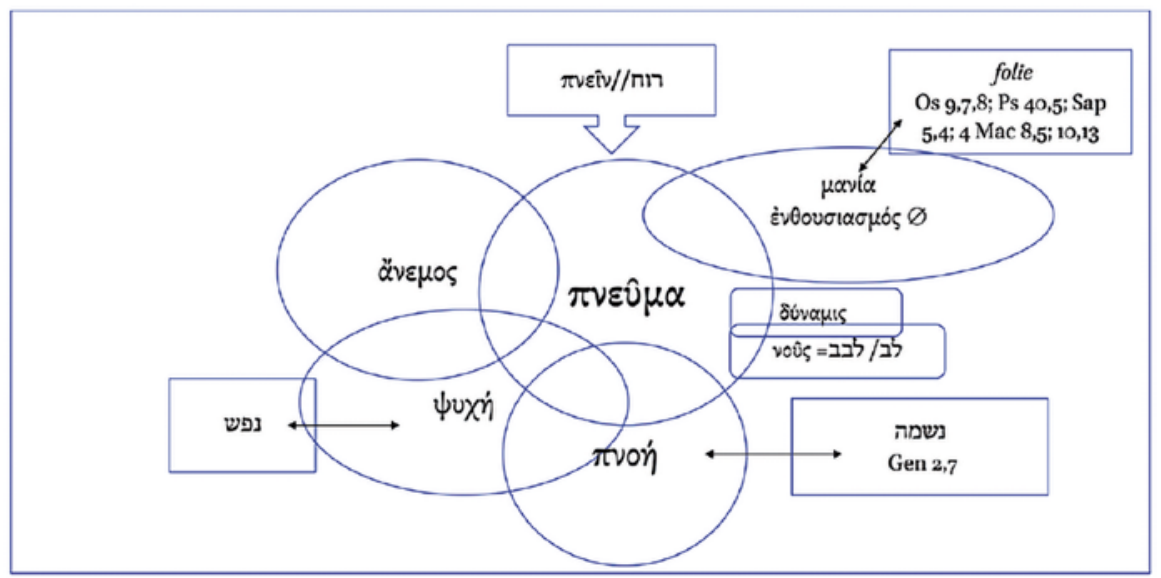

Carte sémantique : $\pi \nu \varepsilon \hat{\mu} \mu \alpha$

transformation du langage qui décrit la possession divine en grec à l'époque hellénistique. Au contraire, dans ce cadre, la Lxx peut être considérée comme l'un des plus anciens témoignages d'un nouveau vocabulaire de la mantique à l'époque hellénistique, lorsqu'une nouvelle réflexion sur la nature de l'inspiration prophétique se met en place. En ce sens, l'apport du dialogue avec les traditions philosophiques grecques serait à reconsidérer, notamment pour les traditions les plus philosophiques de la LXX, telles que, par exemple, Ben Sira et le livre de la Sagesse, qui montrent l'esprit divin comme une véritable puissance agissante dans le monde, à côté de la Sagesse elle-même ${ }^{104}$.

À la lumière de ces données, j'émettrais en revanche une nouvelle hypothèse. La nouveauté amenée par la LXX dans la conceptualisation du $\pi v \varepsilon \hat{\mu} \mu \alpha$ ne concerne pas tellement l'idée d'un esprit divin, mais plutôt la représentation des $\pi \nu \varepsilon \dot{\mu} \mu \alpha \tau \alpha$ comme puissances «plurielles », à savoir des esprits distingués de la divinité, qui peuvent être nuisibles pour les hommes et apparaissent, de ce fait, très proches des démons. Dans la mesure où cette conceptualisation

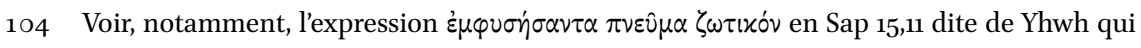
« infuse un souffle vital» dans ses créatures. Les parallèles avec les descriptions grecques de l'extase prophétique ont été également observés par Levison 2009, p. 154-177 qui considère que les auteurs juifs ont consciemment emprunté ce langage grec, défini par Levison comme l'«héritage de Delphes», pour relire les prophéties bibliques. Alors que cette interprétation est possible pour Josèphe et Philon, elle n'est pas nécessaire pour comprendre l'usage de $\pi v \varepsilon v \hat{\mu} \alpha$ dans la LXX. En outre, la construction de la prophétie de Delphes comme provoquée par une possession par l'esprit est une construction tardive dans les sources grecques : la LXx, à cet égard, n'est pas une héritière, mais un antécédent. Pour le rapport de la Sagesse avec le stoïcisme quant à la conceptualisation de l'esprit, voir M. Edwards 2012. 
remonte déjà à la Bible hébraïque, on peut véritablement parler à cet égard d'une influence de l'hébreu rûaḥ sur le grec $\pi \nu \varepsilon \hat{\mu} \mu$. Mais les traditions liées à la LXX développent ultérieurement la représentation des esprits : nous avons déjà vu les changements qui s'opèrent en $1 \mathrm{R} 22$ et en $1 \mathrm{~S} 16$. Nous pouvons encore ajouter quelques données provenant d'autres traditions de la LXX qui montrent des traces d'une progressive autonomisation des esprits par rapport à Yhwh.

\section{Sur quelques développements des mauvais esprits dans la LXX}

Nous avons au moins deux indices supplémentaires dans la LXX qui attestent une progressive autonomisation des esprits. Le premier est d'ordre linguistique, et plus précisément syntaxique. Comme nous l'avons vu plus haut, la formulation qui voit rûah suivi par un substantif de spécification est la manière la

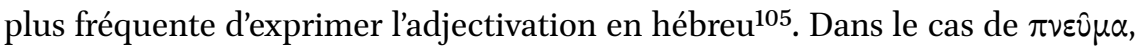
cette construction est reproduite fidèlement dans la plupart des cas en grec, comme le montre le tableau 9 ci-dessous. Elle est également adoptée par les auteurs de langue grecque dont le registre est littéraire et élevé, comme nous pouvons le voir en Sap 7,7. À l'inverse, nous remarquons que les traducteurs choisissent rarement de faire suivre $\pi \nu \varepsilon \hat{u} \mu \alpha$ par un adjectif. Nous retrouvons cette construction seulement deux fois : en $1 \mathrm{R} 22$ pour qualifier l'esprit de mensonge comme « esprit mensonger » et en Zach 13,2 pour qualifier l'esprit d'impureté comme «esprit impur» :

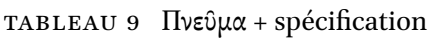

\begin{tabular}{|c|c|c|c|}
\hline Ex 28,3 & $\pi v \varepsilon \dot{\mu} \mu \alpha \tau O \varsigma \alpha i \sigma \theta \dot{\eta} \sigma \varepsilon \omega \varsigma$ & רוח חכמה & Esprit de sagesse \\
\hline Ex 31,3 & 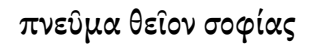 & רוח חכמה & \\
\hline Ex 35,31 & 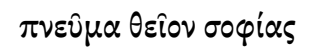 & רוח חכמה & \\
\hline Deut 34,9 & 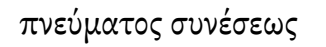 & רוח חכמה & \\
\hline Num 5, 14, 30 & $\pi \nu \varepsilon \hat{\nu} \mu \alpha \zeta \eta \lambda \omega ́ \sigma \varepsilon \omega \varsigma$ & רוח קנאה & Esprit de jalousie \\
\hline $1 \mathrm{R} \mathrm{22,22}$ & 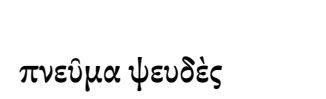 & רוח שקר & Esprit de mensonge \\
\hline Zach 12,10 & 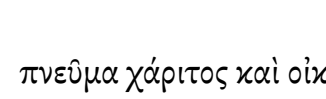 & רוח חן ותחנונים & $\begin{array}{l}\text { Esprit de grâce et de } \\
\text { supplication }\end{array}$ \\
\hline
\end{tabular}

105 Voir Joüon $§ 141$. 


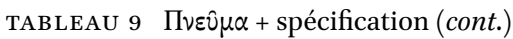

\begin{tabular}{|c|c|c|}
\hline Zach 13,2 & רוח הטמאה & $\begin{array}{l}\text { Esprit d'impureté/ } \\
\text { esprit impur }\end{array}$ \\
\hline Is 4,4 & 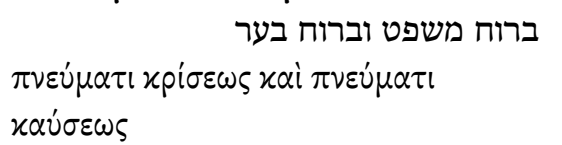 & $\begin{array}{l}\text { Esprit de jugement } \\
\text { Esprit d'incendie/de } \\
\text { dévastation }\end{array}$ \\
\hline Is 11,2 & 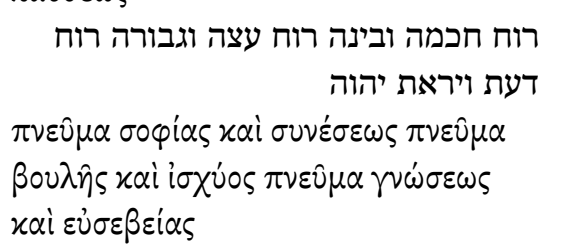 & $\begin{array}{l}\text { Esprit de sagesse, } \\
\text { de discernement, } \\
\text { de conseil, de force, } \\
\text { de connaissance, de } \\
\text { crainte de Yhwh }\end{array}$ \\
\hline Is 29,10 & רוח תרדמה & Esprit de torpeur \\
\hline Os 5,4 & $\pi v \varepsilon \hat{\mu} \mu \alpha \pi \circ \rho v \varepsilon i \alpha \varsigma$ & Esprit de prostitution \\
\hline Sap 7,7 & 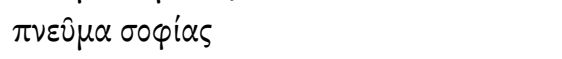 & Esprit de sagesse \\
\hline Sir 39,6 & 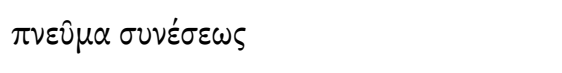 & Esprit d'intelligence \\
\hline \multicolumn{3}{|c|}{ 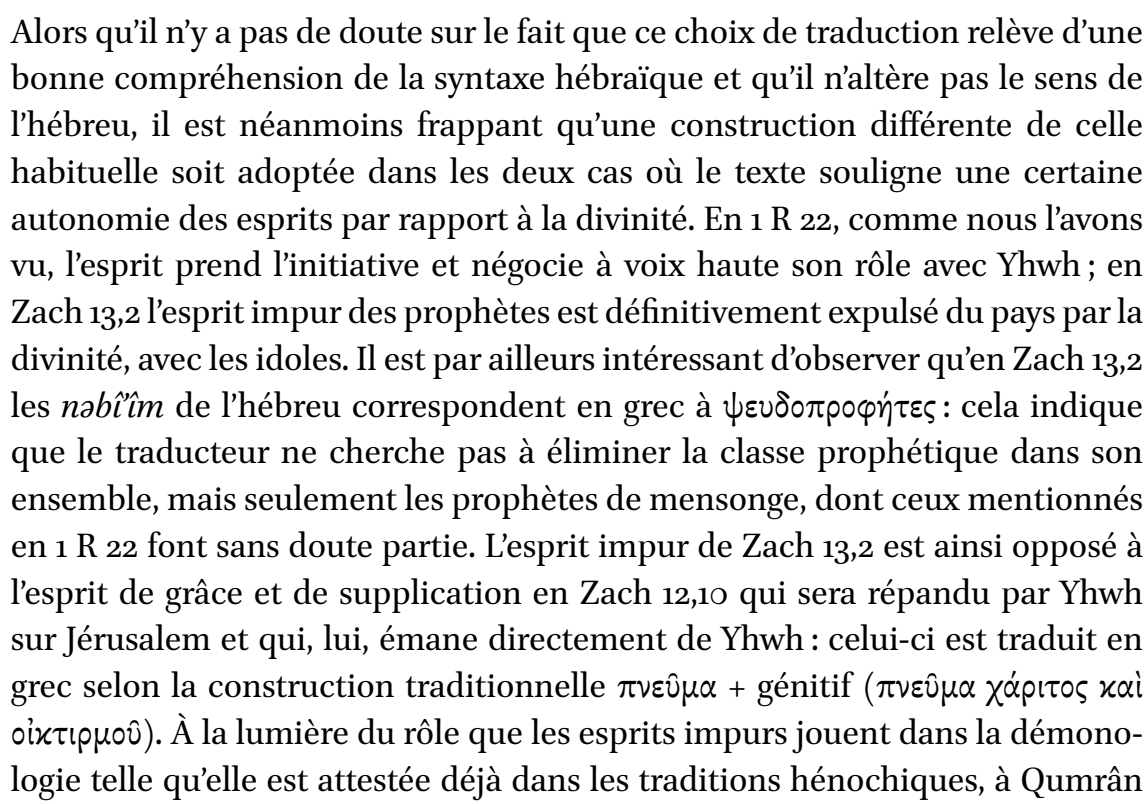 } \\
\hline
\end{tabular}


ainsi que dans le Nouveau Testament ${ }^{106}$, nous pouvons nous demander si le traducteur de Zacharie ne vise pas à séparer intentionnellement cet esprit, qui reste mal contrôlé par Yhwh, des autres esprits qui sont en lien plus étroit avec lui. Cette démarche serait d'ailleurs en lien avec la tendance des traditions grecques à distinguer plus nettement les esprits divins des esprits mauvais,

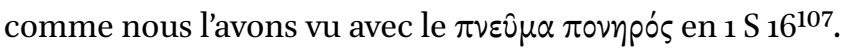

La deuxième trace d'une relative autonomisation des esprits et d'une proximité entre esprits et démons est probablement à repérer dans un passage de la Sagesse de Salomon. Au chapitre 7, versets 17-20, Salomon énumère une liste des compétences qu'il a acquises par la divinité. Il s'agit de connaissances que l'on dirait aujourd'hui «scientifiques»: elles concernent les sciences naturelles, qui comprennent l'astrologie, la zoologie, l'anthropologie, et la médecine :

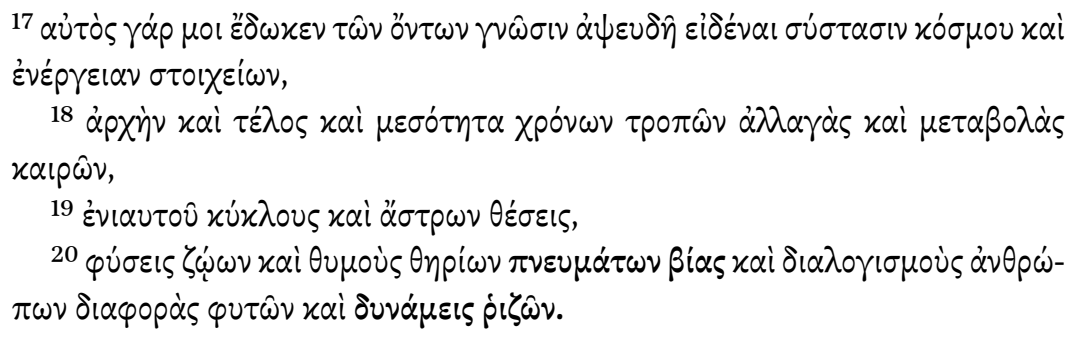

${ }^{17}$ Ainsi m’a-t-il donné une connaissance parfaite du réel. Il m’a appris la structure de l'univers et l'activité des éléments,

${ }^{18}$ le commencement, la fin et le milieu des temps, les alternances des solstices et les changements de saisons,

${ }^{19}$ les cycles de l'année et les positions des astres,

${ }^{20}$ les natures des animaux et les tempéraments des bêtes sauvages, les violences des esprits et les pensées des hommes, les variétés de plantes et les vertus des racines.

106 Pour une interprétation démonologique de l'esprit d'impureté dans les traditions du Second Temple, voir Lange 2003.

107 Il est, en revanche, remarquable que le traducteur grec des Nombres n'ait pas interprété dans le même sens l'esprit de jalousie en Num 5,14 et 3o. À cet égard, il faut observer qu'aucune interprétation « démonologique » ne paraît attestée pour cet esprit dans la littérature du Second Temple: dans les écrits de Qumrân l'expression רנאה nנאה napparait jamais dans les catalogues d'esprits, qui sont pourtant assez nombreux. Elle apparaît une fois dans les Hodayot, mais avec une signification positive ( $1 \mathrm{QH}^{\mathrm{a}} 10,17$ : « un esprit zélé »). 
Dans ce passage la question se pose quant à la manière dont il faut comprendre $\pi \nu \varepsilon u \mu \alpha \dot{\tau} \tau \omega \nu$. Alors que la plupart des chercheurs modernes le traduisent par « esprits », Giuseppe Scarpat ${ }^{108}$ a repéré un bon nombre d'exemples dans la littérature grecque et latine où l'expression $\beta i ́ \alpha \pi \nu \varepsilon v \mu \alpha \dot{\alpha} \tau \omega \nu / v i s$ ventorum se réfère à la «violence des vents ». Par conséquent, il suggère d'entendre $\pi \nu \varepsilon u ́ \mu \alpha \tau \alpha$ ici comme «vents » : cette interprétation trouverait sa place dans la description de phénomènes naturels tels que le cycle des saisons et la position des astres. Toutefois, il me semble que la référence aux esprits est la plus probable en ce contexte, non seulement car le thème de la violence des esprits est bien connu dans les traditions du Second Temple ${ }^{109}$, mais également pour deux raisons structurelles. Premièrement, la description des domaines de compétence du roi Salomon en ce passage suit une progression structurée : l'on passe des éléments les plus généraux qui composent l'univers (verset 17), aux phénomènes météorologiques et astrologiques (versets 18-19), aux animaux (verset 20 :

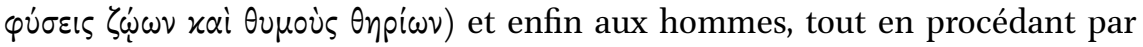
couples de deux exemples chacun. Il me semble donc que les $\pi v \varepsilon u \mu \alpha \dot{\tau} \tau \nu \nu \beta i \alpha \varsigma$ du verset 20 ne renvoient pas aux phénomènes atmosphériques mentionnés plus haut au verset 17 , mais doivent être mis en relation avec les raisonnements

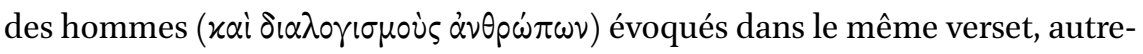
ment dit cette expression semble décrire un phénomène qui a à voir avec les hommes. Un autre argument en faveur de cette lecture, et j'en viens ici à ma deuxième raison, provient également de la référence suivante aux plantes et

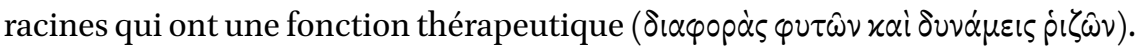
Comme nous le verrons par la suite, l'usage de remèdes végétaux dans un but apotropaïque est bien attesté dans la LXX, notamment dans le livre de Tobit. Si cette interprétation est correcte, nous aurions ici une trace de l'émergence du personnage de Salomon à côté de David comme figure capable de maîtriser démons et esprits ; figure qui aura un succès immense dans les traditions successives. À cet égard, la LXX ne représente pas un cas isolé, mais s'inscrit dans plusieurs traditions sur la figure de Salomon qui se développent à partir de l'époque du Second Temple ${ }^{110}$.

Il faut toutefois préciser que l'autonomie des esprits mauvais dans la LXX et leur caractérisation négative n'impliquent pas encore nécessairement l'adoption d'une cosmologie strictement dualiste où un groupe d'esprits malveillants serait opposé à l'armée céleste de Yhwh. Au contraire, tous ces esprits

\footnotetext{
108 Scarpat 1996, p. $5^{2-56 .}$

109 Notamment dans le mythe des Veilleurs du livre d'Hénoch ainsi que dans les Jubilés, voir par exemple VanderKam 2003 ; A.T. Wright 2005 ; Barbu et Rendu-Loisel 2009, p. 331-338, Brand 2013, p. 173-179.

110 La littérature sur le sujet est abondante : une bonne introduction est sans doute Torijano 2002.
} 
demeurent sous l'influence ultime de la divinité, même si leur degré d'agentivité et, par conséquent, leur dangerosité sont plus prononcés que chez d'autres membres de la cour céleste et plus explicites dans la LXX que dans la Bible hébraïque. D'ailleurs la Lxx ne donne pas une interprétation univoque des esprits. À côté de cette tendance à une « démonisation » des esprits, une tendance inverse, voire diamétralement opposée, est également attestée dans la LXX, qui fait des $\pi v \varepsilon \dot{\mu} \mu \alpha \tau \alpha$ des créatures angéliques. C'est donc vers le rapport entre esprits, anges et démons qu'il faudra diriger notre attention. Avant de passer à une analyse plus détaillée de ces relations, je souhaite néanmoins conclure par un cas de figure particulier dans le livre de Job, où il est question d'une vision spectrale apparue à son ami Eliphaz.

Apparitions nocturnes (Job 4,13-16)

Le récit de la vision d'Eliphaz, qui rend visite à son ami Job pour le soulager de ses disgrâces, couvre les versets 12-21 du chapitre 4 du livre. Le texte hébreu a fait l'objet d'un certain nombre de discussions. Le caractère non conventionnel de la révélation faite par une voix - vraisemblablement divine - à Eliphaz, aux contenus très pessimistes sur la nature divine et sur le destin humain, a amené quelques chercheurs à émettre l'hypothèse que la vision n'aurait à l'origine pas été attribuée à Eliphaz, mais qu'elle concluait la lamentation de Job au chapitre 3 et qu'elle aurait ensuite été déplacée ${ }^{111}$. La Septante ancienne, avec le reste de la tradition antique, conserve toutefois le passage à la même position que celle où il se trouve dans le texte massorétique. Parmi les points les plus débattus du texte se trouve, notamment, l'interprétation du rûah (ici au masculin) qui passe sur le visage d'Eliphaz et sert de vecteur de la révélation divine :

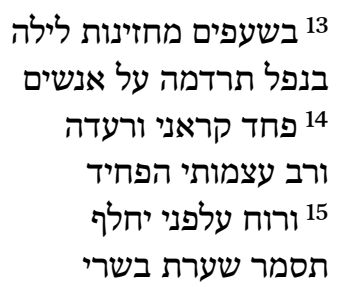

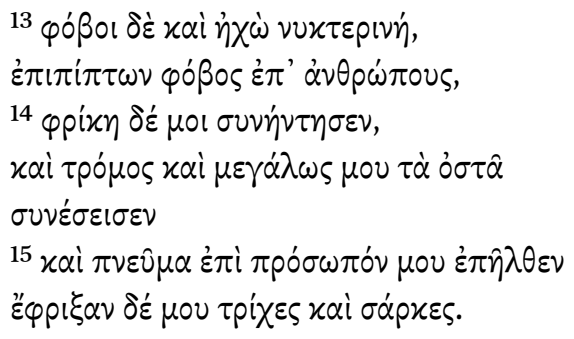

111 Cette proposition a été récemment reprise par Ken Brown (2015), auquel je renvoie pour toute référence bibliographique sur la question. 


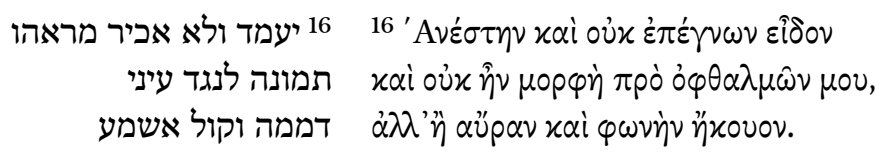

${ }^{13}$ Dans les pensées troublantes des visions nocturnes, quand une torpeur tombe sur les hommes, ${ }^{14}$ une peur m'a surpris, et un frisson, et il a fait trembler tous mes os, ${ }^{15}$ un esprit/souffle passa sur ma face, visage, les poils de ma chair frissonnèrent. ${ }^{16}$ Il se tenait debout, je ne reconnus pas son aspect, une forme était devant mes yeux. Silence, puis j'entendis une voix.
${ }^{13}$ Les terreurs et une rumeur nocturne, peur qui s'abat sur les hommes, ${ }^{14}$ un frisson et un tremblement m'ont saisi,

et ils ont secoué avec force mes os ${ }^{15}$ et un esprit/souffle passa sur mon et les poils de ma chair frissonnèrent. ${ }^{16} \mathrm{Je}$ me levai, et je ne le reconnus pas, je regardai, et il n'y avait pas de forme devant mes yeux, rien qu'un mouvement d'air, et j'entendis une voix.

L'interprétation moderne est partagée quant à la compréhension de ce rûah : certains le traduisent par «esprit», tandis que d'autres préfèrent une traduction par « vent », ou « souffle »112. À cet égard, une tension est souvent observée entre les versets 15 et 16 : l'expression 'al pānay yahălōp («passa sur ma face», verset 15 a) n'est jamais utilisée ailleurs pour les esprits, mais le verbe $h l p$, «passer », est employé lorsqu'il est question de vents ou d'autres tourbillons naturels, par exemple en Is 21,1 et Hab 1,11. Parallèlement, $h l p$ décrit le passage de la divinité dans le livre de Job ${ }^{113}$. En outre, au verset 16 la présence du verbe ' $m d$, «se tenir debout», qui requiert un sujet et apparaît également en $1 \mathrm{R} 22$, semble indiquer qu'il vaut mieux comprendre rûah comme un esprit, dont Eliphaz ne verrait que les contours (təmûnâ), sans en reconnaitre l'identité. À cet égard, on parle souvent pour ce passage d'ambiguïté intentionnelle

\footnotetext{
112 «Esprit»: B. Duhm 1897 ; Hartley 1988, p. 108-109; Brown 2015, p. 75-76; «vent » : Clines 1989, p. 130 ; Seow 2013, p. 401 ; « souffle »: Terrien 1963, p. 71. Il faut également signaler que l'interprétation de śăărâ, «poil», au verset 15 est également débattue car le mot est parfois considéré comme une graphie alternative de səāâa, «tourbillon », « ouragan », terme fréquent dans le contexte de théophanies (Job 38,1; 40,6). Je suis d'accord avec Brown (2015, p. 76) sur le fait que l'image des poils qui se hérissent est ici l'interprétation la plus naturelle et la mieux adaptée au contexte ; en outre, elle a l'appui d'un certain nombre de parallèles mésopotamiens : voir Paul 1983.

113 Job 9,11; 11,10.
} 
ou de « double sens » ${ }^{114}$ : même les chercheurs qui comprennent rûah comme «vent» admettent que le texte fasse en même temps allusion à la manifestation d'un élément surnaturel ${ }^{115}$. Il me semble que la tension peut être résolue par une lecture indexicale du rûah :indépendamment du choix de traduction par «souffle » ou « esprit», rûah indique ici le mouvement d'air qui est le signe perceptible de la présence d'une puissance surnaturelle et dont le passage produit un effet tangible sur le corps d'Eliphaz (les poils de sa peau se hérissent). En revanche, il est à mon avis moins sûr de savoir s'il faut interpréter cet esprit comme un esprit mensonger à l'instar de 1 R 22, comme le suggèrent Harding et Hamori, car le contexte n'est pas celui de la prophétie ni de l'assemblée divine. Il est plutôt fait référence au langage des théophanies qui est repris et, en même temps, déformé par le rédacteur par son insistance sur les aspects terrifiants de la vision ${ }^{116}$. Il faut également noter que la nature divine de la vision et de la voix qui parle à Eliphaz n'est nulle part mentionnée, ce qui soulève, probablement de manière intentionnelle, des doutes légitimes sur l'origine de l'esprit ${ }^{117}$.

Lorsque l'on regarde le choix du traducteur grec, on remarque que le double sens «souffle/esprit» ainsi que l'aspect sensoriel de la vision sont parfaitement préservés par $\pi v \varepsilon \hat{\mu} \alpha \alpha$ et renforcés par l'équivalence entre dəmāmâ (litt.

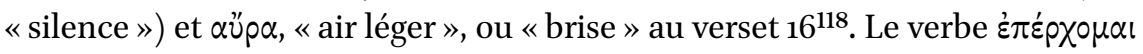
n'est pas un équivalent habituel pour $h l p$, mais il est utilisé pour décrire l'action de l'esprit de jalousie en Num 5,14 et 30, et ailleurs dans le livre de Job pour des phénomènes naturels violents comme des vents ou des tourbillons ${ }^{119}$ : il se prête donc à une double lecture. En outre, le grec atteste une série de changements supplémentaires qui ont comme conséquence principale le fait que l'aspect proprement visuel de l'apparition a complètement disparu du texte. Le verbe $\alpha \dot{v i} \sigma \tau \eta \mu$, équivalent de ' $m d$, est à la première personne et le substantif mar'ê, « apparence», est rendu par le verbe عîंov, «je regardai ». Eliphaz se

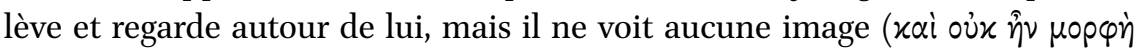
$\pi \rho \dot{o} \partial \varphi \theta \alpha \lambda \mu \hat{\omega} \nu \mu \circ v)$. Si le changement de la personne du verbe et le choix de

114 Fullerton 1930 ; Harding 2005.

115 Voir Seow, p. 40.

116 Harding 2005, p. 149-152 ; Hamori 2010, p. 24-26. Le contenu de la révélation (vv. 17-21) bien que pessimiste, ne peut pas être qualifié simplement de faux ou trompeur car l'idée que la divinité n’a pas à cœur les destins humains est un des piliers traditionnels de la réflexion sapientielle, biblique et proche-orientale plus largement : voir sur ce point, par exemple, Witte 1994, p. 98-110.

117 D'où la suggestion, parfois proposée, de voir Satan à l'œuvre derrière la voix qui parle à Eliphaz : voir, à ce sujet, la critique de Brown 2015, p. 19-21.

118 Il s'agit d'une équivalence régulière dans la LXX : comparer avec 3 Regn 19,12 (= TM 1 R 19,12); Ps 106,29 (= TM 107,29).

119 Job 1,19;37,9. 
traduire mar'ê par عî̉ov sont vraisemblablement imputables au traducteur ${ }^{120}$,

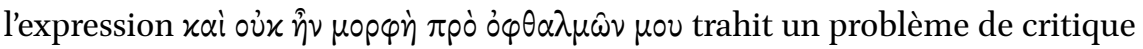
textuelle. Comme l'observe Seow ${ }^{121}$, le texte grec s'explique facilement par une division différente du texte consonantique : il semble que le traducteur ait lu m'rh wtmwnh l'ngd 'yny, au lieu de m'rh wtmwnh lngd 'ymy. La Peshitta a ici une version simplifiée, sans équivalent pour təmûnâ, mais avec la négation («je me levai et je ne reconnus pas, et il n'y avait rien à voir devant mes yeux»). Les traditions latines conservent les deux versions: alors que la Vulgate est en accord avec le texte massorétique, d'autres témoins de la Vieille Latine ont un texte proche de la LXX ancienne ${ }^{122}$. L'ensemble des témoins manuscrits fait pencher vers l'hypothèse selon laquelle le changement ne serait pas imputable au traducteur, mais qu'il serait survenu à un certain stade de la transmission du texte hébreu. Cette correction harmonise la deuxième moitié du verset 16 avec sa première moitié où Eliphaz affirme qu'il ne reconnaît pas l'image devant lui : de ce point de vue le texte massorétique est supérieur en tant que lectio difficilior. La version préservée par la LXX atteste, donc, une tradition ancienne qui transforme l'expérience sensorielle de la vision d'Eliphaz qui était, pour ainsi dire, à 36o degrés, en une expérience exclusivement auditive et tactile. D'un côté, comme ceci a déjà été remarqué par Clines, cette tradition s'inscrit facilement dans un contexte exégétique où la référence à des images divines pouvait être problématique ${ }^{123}$. D'un autre côté, elle ajoute, néanmoins, un nouvel élément à la représentation des esprits, qui sont ici décrits comme des entités invisibles à l'œil humain, voire franchement immatérielles : ils ne sont rien d'autre qu'« air et voix ».

120 Je ne pense pas qu'il soit nécessaire de postuler un originaire عî̉oৎ, «forme», «appa-

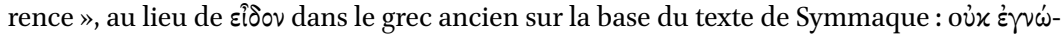

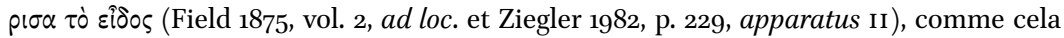
a été proposé par Katz (1947, p. 195) sur la base d'une indication qui remonte à Johann Friedrich Schleusner. Mar'ê ne signifie pas seulement « apparence », mais indique parfois l'acte de voir : il me semble qu'عî̉ov résulte de la volonté du traducteur de rendre explicite l'effort fait par Eliphaz de chercher autour de lui le responsable du frissonnement qu'il ne peut pas reconnaître. En revanche, le texte de Symmaque s'explique bien comme une correction du grec faite sur la base du texte massorétique.

121 Seow 2013, p. 402-403.

122 Clem. R., Ep. 39 : et non erat forma ante oculos meos; Jer., Interpr.Job. (ed. Martianay, Migne, PL 29, 69): Inspexi : et non erat figura ante faciem meam; Augustinus, Annotationes Job ad loc. (PL 34): nam negat in consequentibus fuisse figuram.

123 Clines, 1989, p. 131. 